\title{
A Model of Numerical Calculation of Conductivity for III-V MBE Epilayers Using a Hall Device
}

\author{
Andrzej Wolkenberg ${ }^{1,2}$ \\ ${ }^{1}$ Institute of Electron Technology, Aleja Lotnikow 32/46, 02-668 Warsaw, Poland \\ ${ }^{2}$ Institute of Materials Engineering, Czestochowa University of Technology, Aleja Armii Krajowej 19, 42-200 Czestochowa, Poland \\ Correspondence should be addressed to Andrzej Wolkenberg; wolkenberg@neostrada.pl
}

Received 13 November 2012; Accepted 10 January 2013

Academic Editor: Ram Katiyar

Copyright (c) 2013 Andrzej Wolkenberg. This is an open access article distributed under the Creative Commons Attribution License, which permits unrestricted use, distribution, and reproduction in any medium, provided the original work is properly cited.

\begin{abstract}
An electrical conduction versus temperature model using a Hall device was developed. In the case of InAs, InGaAs, and GaAs MBE epilayers, the prediction agrees well with the experimental results. Herein, we explain here how these calculated fractions of total conductivity describe the measured values. The method allows for the calculation of the carrier concentration and mobility of each component of a multicarrier system. The extracted concentrations are used to characterise the different components of charge transport in the active layer. The conductance values $G$ [S] of these components of charge transport were obtained. Also the scattering events for the investigated samples are presented. The analysis of the experimental results for three semiconductor compositions and different concentrations demonstrates the utility of our method in comparing the conductance of each component of the multilayered system as a function of temperature.
\end{abstract}

\section{Models}

1.1. Two Layer Model. We take as an example a physical twolayer model according of thin InAs MBE layers. After etching step by step Grange et al. [1] obtained a $1 \mu \mathrm{m}$ InAs MBE layer with the concentration profile shown in Figure $2(\boldsymbol{\nabla})$. Near the interface with the GaAs substrate, the researchers measured the concentration to be as high as $n=1 \cdot 10^{18} / \mathrm{cm}^{3}$.

This result is in agreement with the TEM image presented in Figure 1 obtained by Fawcett et al. [2]. We proposed that the MBE layer of InAs consists of two parts:

(i) a strongly defected layer measuring $t_{1}$ in thickness,

(ii) a correctly formed layer measuring $t_{2}-t_{1}$ in thickness, where $t_{2}$ is the metallurgical thickness.

We present in Figure 2 data regarding the concentration values obtained by many authors. The following equation is used to relate the experimental concentration data with the InAs layer thickness:

$$
n=\frac{n_{1} \cdot t_{1}+n_{2} \cdot\left(t_{2}-t_{1}\right)}{t_{2}}
$$

where $n_{1}=2 \cdot 10^{19} \mathrm{~cm}^{-3}, t_{1}=18.2 \cdot 10^{-8} \mathrm{~cm}$, and $n_{2}=$ $1 \cdot 10^{15} \mathrm{~cm}^{-3}$.

Therefore one can convince one's self that the two-layer model may be practically used in our calculation.

1.2. Calculation Background. Electron and hole concentrations are derived by the numerical solution of the neutrality equation:

$$
n_{e}+x_{e, h}=p+N_{D 1}^{+}+N_{D 2}^{+}-N_{A}^{-} \text {, }
$$

where $n=n_{e}+x_{e, h}$ is the total conductivity electron concentration,

$$
x_{e, h}=x_{D}-\sqrt{\frac{x_{A} N_{V}}{2}} \exp \left[-\frac{E_{X, A}}{k T}\right]\left[\mathrm{cm}^{-3}\right],
$$

$x_{e, h}$ is the net concentration, $x_{D}$ is the concentration of the donor-like states, $x_{A}$ is the concentration of the acceptor like state, $N_{V}$ is the effective density of states in the valence band, $E_{X, A}$ is the ionisation energy of the acceptor-like states, $k$ is the Boltzmann constant, $p$ is the hole concentration, $N_{D 1}^{+}$is the concentration of shallow donors with activation energy 


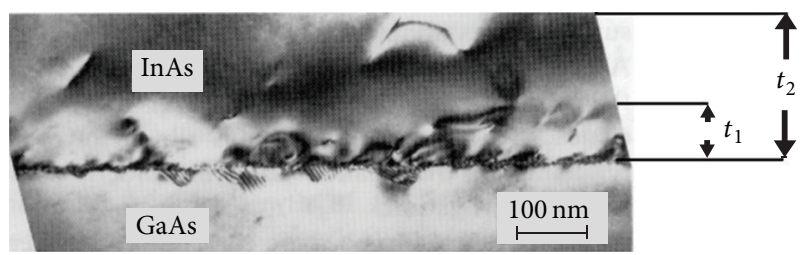

Figure 1: Two-layer pattern of MBE InAs layer after Fawcett et al. [2] and our interpretation: $t_{1}$-thin interface layer measuring $1.82 \mathrm{~nm}$ in thickness with high charge carrier concentration $n_{1}$ and high FWHM value; $\left(t_{2}-t_{1}\right)$-thick bulk layer whose thickness is the difference between the metallurgical thickness $t_{2}$-and defected layer thickness $t_{1}$ with mean charge carrier concentration $n_{2}$.

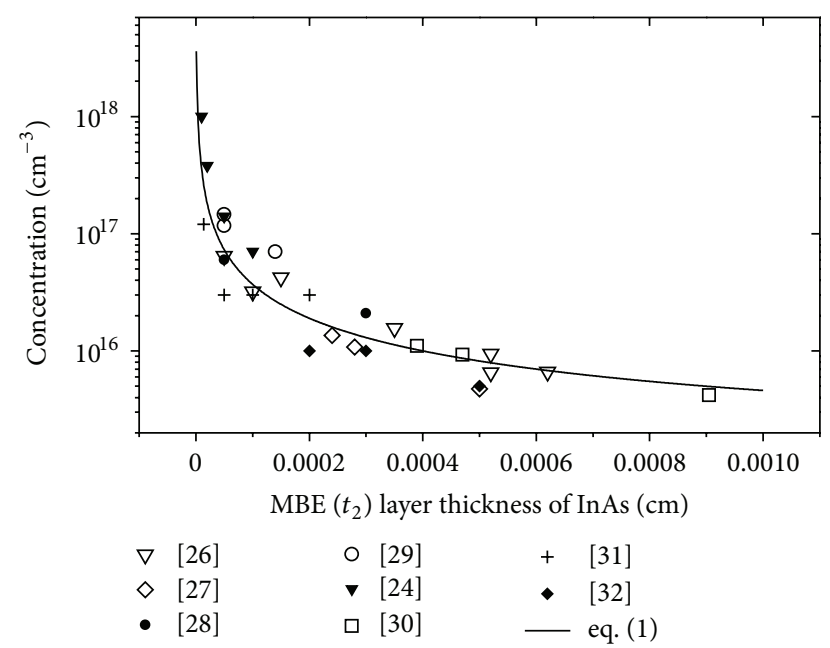

FIGURE 2: Charge carrier concentration versus thickness of $t_{2}$ layer of InAs deposited at $500^{\circ} \mathrm{C}$ on GaAs substrate.

$E_{D 1}, N_{D 2}^{+}$is the concentration of deep donors with activation energy $E_{D 2}$, and $N_{A}^{-}$is the concentration of acceptors with activation energy $E_{A}$.

To obtain the mobility, we numerically integrate the equation

$$
\begin{aligned}
\frac{1}{\tau_{n, h}}= & \frac{1}{\tau_{\mathrm{ii}(n, h)}}+\frac{1}{\tau_{\mathrm{dp}(n, h)}}+\frac{1}{\tau_{\mathrm{pie}(n, h)}} \\
& +\frac{1}{\tau_{\mathrm{po}(n, h)}}+\frac{1}{\tau_{\mathrm{sc}(n, h)}}+\frac{1}{\tau_{\operatorname{dis}(n, h)}}\left[\mathrm{s}^{-1}\right],
\end{aligned}
$$

where the number of used $\tau$ values depends on values presented in Tables 4,8 , and 12 and the scatterings times $\tau$ are presented in Table 1 .

\section{Introduction}

Structural inaccuracy, especially in layered structures, is one factor that affects how the magnitude of a magnetic field influences measured charge transport properties [3]. While studying the transport properties of HgCdTe layers, Gold and Nelson [4] reported on the influence of different charge

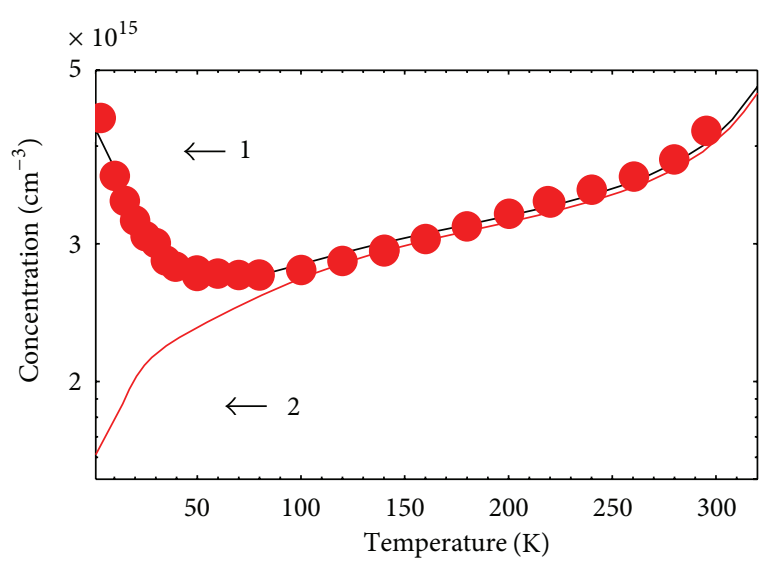

Figure 3: Concentration versus temperature $n$-InAs $9.0 \mu \mathrm{m} ; N_{A}=$ $8.05 \cdot 10^{15} \mathrm{~cm}^{-3}, N_{D 1}=1.26 \cdot 10^{16} \mathrm{~cm}^{-3} ; N_{D 2}=8.5 \cdot 10^{15} \mathrm{~cm}^{-3} ; E_{A}=$ $0.005 \mathrm{eV} ; E_{D 1}=0.001 \mathrm{eV} ; E_{D 2}=0.13 \mathrm{eV} ; x_{A}=4.2 \cdot 10^{15} \mathrm{~cm}^{-3} ; x_{D}=$ $1.2 \cdot 10^{15} \mathrm{~cm}^{-3} ; E_{x_{A}}=0.0008 \mathrm{eV}$; red bullets: $n_{H}$ experimental data, (1) black line: $n$ calculated curve; (2) red line: $n_{H}$ calculated curve [3].

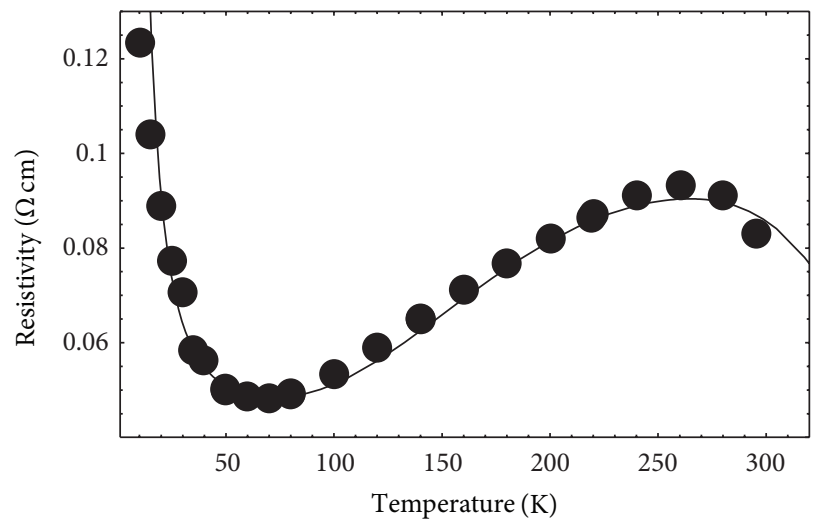

FIgURE 4: The resistivity versus temperature semiconductor parameters as in Figure 3, with $\mu$ calculated by scattering on ionized impurities, acoustic phonons with deformation potential, acoustic phonons with piezoelectric potential, polar optical phonons and space charge with $N_{S} \cdot Q=0.03, \cdots \rho$ experimental points, black line: $\rho$ calculated curve (e-electron charge).

carriers using Hall characterisation. An exhaustive review concerning these phenomena was undertaken by Meyer et al. [5]. Such disorder was first described and interpreted by the so-called mobility spectrum by Beck and Anderson [6] and many papers have focused on this problem [7-20]. For the proper characterisation of a multicarrier conduction system, the number of independent measurements must match the number of desired physical quantities. In actuality, the complete characterisation of multicarrier conduction is extremely complex. Even for two-carrier conduction, one must make four independent measurements to calculate the individual carrier concentration and mobility of each component [21]. To this end, other methods, such as the maximum entropy approach [22] or the application of Bryan's algorithm [23], must be considered. The concept most often used in the 


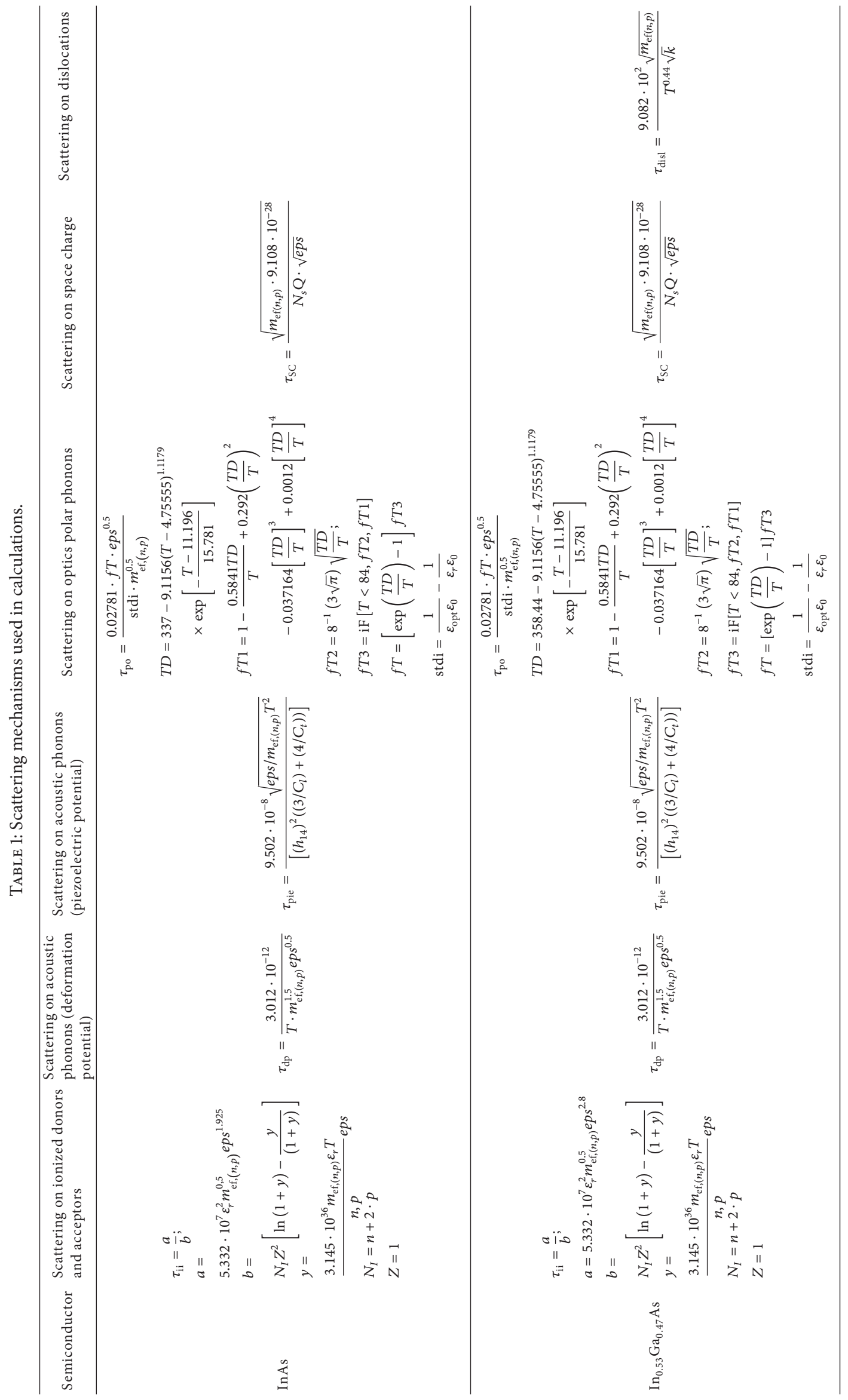




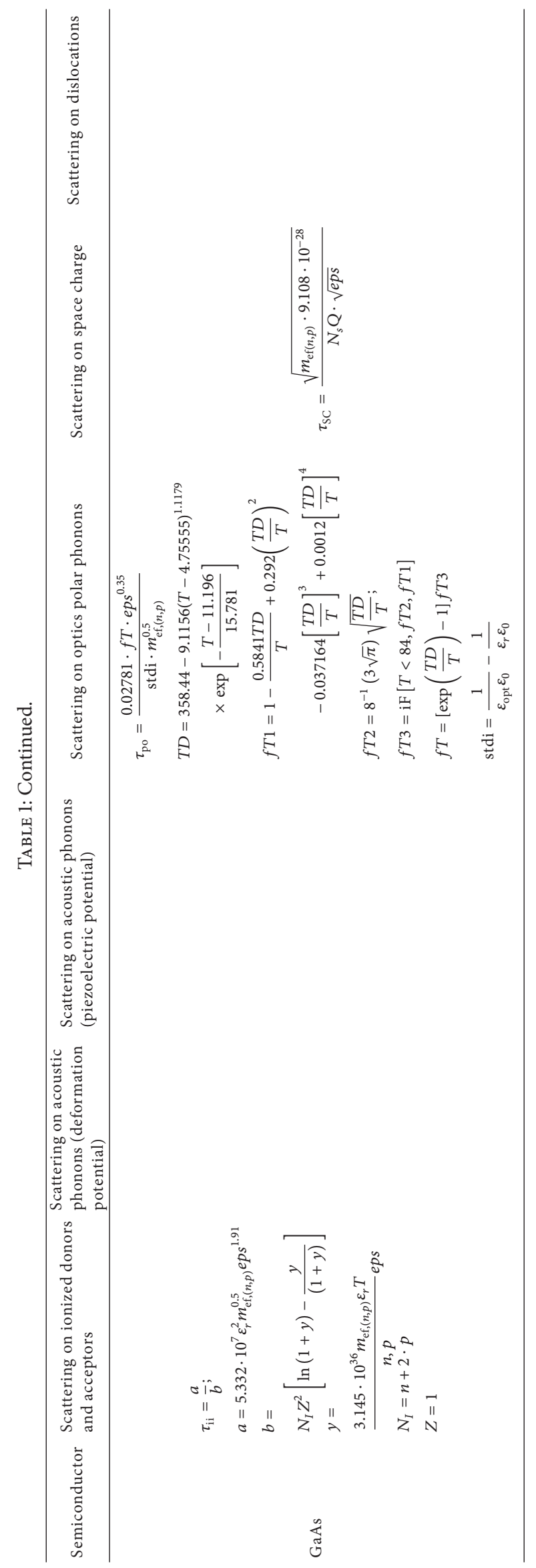


TABLE 2: Main properties of the investigated semiconductors at $300 \mathrm{~K}$.

\begin{tabular}{|c|c|c|c|c|c|c|}
\hline $\begin{array}{l}\text { Investigated } \\
\text { semiconductor }\end{array}$ & $\begin{array}{c}\text { Energy gap } \\
{[\mathrm{eV}]}\end{array}$ & $\begin{array}{c}\text { Intrinsic } \\
\text { concentration } \\
{\left[\mathrm{cm}^{-3}\right]} \\
\end{array}$ & $\begin{array}{c}\text { Average distance } \\
\text { between impurities } R_{d} \\
{[\AA]}\end{array}$ & $\begin{array}{c}\text { Donor state } \\
\text { Bohr radius } a_{B} \\
{[\AA]}\end{array}$ & $R_{d} / a_{B}$ & $\begin{array}{c}\text { Donor state } \\
\text { ionisation energy } E_{B} \\
{[\mathrm{meV}]}\end{array}$ \\
\hline InAs & 0.354 & $\sim 1 \cdot 10^{15}$ & 384.5 & 367 & 1.05 & 1.36 \\
\hline $\mathrm{In}_{0.53} \mathrm{Ga}_{0.47} \mathrm{As}$ & 0.743 & $8.5 \cdot 10^{11}$ & 1224.6 & 180 & 6.8 & 2.8 \\
\hline GaAs & 1.43 & $2.2 \cdot 10^{6}$ & 809.5 & 104 & 7.8 & 5.25 \\
\hline
\end{tabular}

TABLE 3: Growth conditions of InAs layers on (001) GaAs substrates.

\begin{tabular}{lccc}
\hline $\begin{array}{l}\text { No. of } \\
\text { procedures }\end{array}$ & $\begin{array}{c}\text { Thickness } \\
{[\mu \mathrm{m}]}\end{array}$ & $\begin{array}{c}\text { Relation between } \\
\text { V/III }\end{array}$ & $\begin{array}{c}\text { Substrate temperature } \\
{\left[{ }^{\circ} \mathrm{C}\right]}\end{array}$ \\
\hline 92 & 4 & 5 & 440 \\
298 & 5.6 & 4.5 & 450 \\
301 & 4.7 & 3.7 & 500 \\
330 & 9.05 & 5.8 & 506 \\
\hline
\end{tabular}

TABLE 4: Scattering mechanism involved in calculation of InAs samples properties.

\begin{tabular}{|c|c|c|c|c|c|}
\hline No. & Parameter & $\begin{array}{l}\text { InAs } 330 \\
9.0 \mu \mathrm{m}\end{array}$ & $\begin{array}{c}\text { InAs } 301 \\
4.7 \mu \mathrm{m}\end{array}$ & $\begin{array}{c}\text { InAs } \\
298 \\
5.6 \mu \mathrm{m}\end{array}$ & $\begin{array}{c}\text { InAs } \\
292 \\
4 \mu \mathrm{m}\end{array}$ \\
\hline & $\begin{array}{l}\text { Mobility } \\
{\left[\mathrm{cm}^{2} / \mathrm{V} \cdot \mathrm{s}\right]}\end{array}$ & $\begin{array}{c}\tau_{\mathrm{ii}}, \tau_{p}, \tau_{\mathrm{po}}, \\
\tau_{\mathrm{pie}}, \tau_{\mathrm{sc}}\end{array}$ & $\begin{array}{c}\tau_{\mathrm{ii}}, \tau_{\mathrm{dp}}, \tau_{\mathrm{po}} \\
\tau_{\mathrm{pie}}, \tau_{\mathrm{sc}}, \tau_{\mathrm{disl}}\end{array}$ & $\tau_{\mathrm{ii}}, \tau_{\mathrm{disl}}$ & $\tau_{\mathrm{ii}}, \tau_{\mathrm{disl}}$ \\
\hline
\end{tabular}

characterisation of mobility spectra has been described in many papers by Antoszewski and collaborators [12, 24, 25]. The main features of the above-mentioned methods are as follows.

(1) The Hall voltage of the investigated semiconductor is not proportionally dependent on the magnetic field.

(2) Such behaviour in the conductivity process results in charge carriers (electrons and holes) with different mobilities.

(3) The mentioned authors $[6,12,24,25]$ used different mathematical approaches to obtain mobility spectra from mobility and conductivity data.

In cases in which this concept is applicable, the calculations are supported by the conductivity tensor dependence on the magnetic field [6]:

$$
\widehat{\sigma}(H) \equiv \sigma_{x x}(H)+i \sigma_{x y}(H)=\int_{-\infty}^{\infty} \frac{s(\mu)(1+i \mu H)}{1+(\mu H)^{2}} d \mu,
$$

where $s(\mu)=S^{+}(\mu) \rightarrow \mu>0$; or $s(\mu)=S^{-}(|\mu|) \rightarrow$ $\mu<0$ and $S^{+}(\mu)$ and $S^{-}(\mu)$ are a pair of conductivity density functions.

Equation (5) allows for the treatment of the nonlinear magnetic field dependence on the Hall effect [10]. Many experimental results have been analysed using this method $[8-12,24,25]$. Antoszewski et al. concluded that a major advantage of mobility spectrum analysis (MSA) and quantitative mobility spectrum analysis (QMSA) over other methods

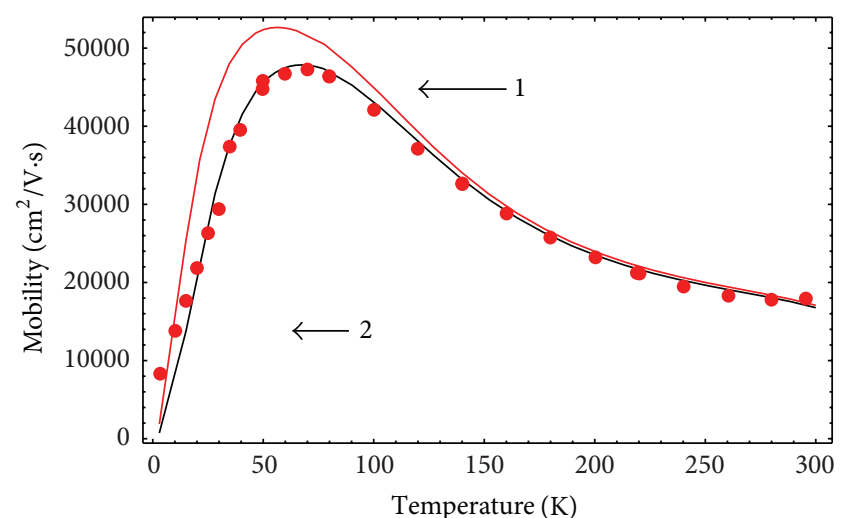

FIGURE 5: Mobility versus temperature calculated using the parameters presented in Figure $3[3] \bullet \cdots \mu_{H}$ experimental points, (1) red line: $\mu_{H}$ calculated curve (see Section 1-Models); (2) (black line) $\mu$ calculated curve (see Section 1-Models).

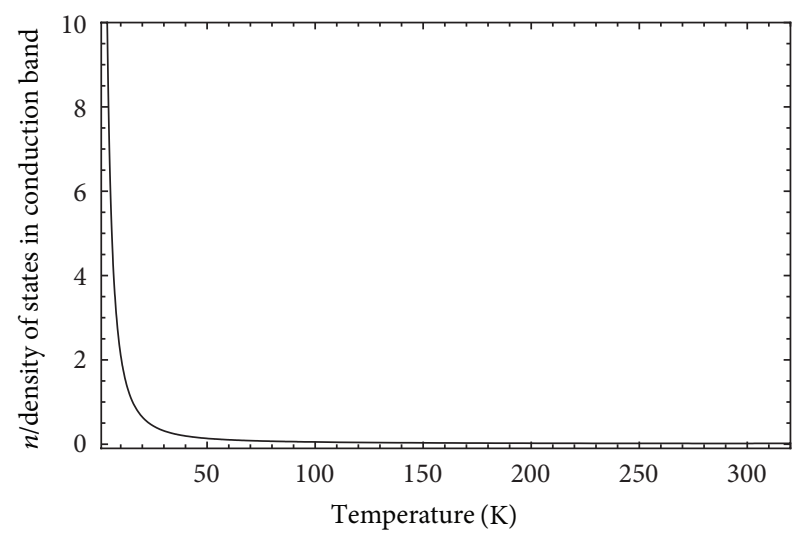

Figure 6: The ratio of electron concentration to density of states in the conduction band versus temperature for the structure shown in Figure 1.

is that these procedures are inherent not arbitrary that is, no prior assumptions are required $[24,25]$. These investigators suggested that their improved (QMSA) method has sufficient reliability, versatility, and sensitivity for their proposed analysis which is fully computer-automated, using magnetic field dependence data as input.

Our approach accounts for several sources of charge carriers as components contribution to the total conductivity of a semiconductor layer [3]. These sources form the parts used to solve the neutrality equation, and the solution is obtained by numerical computer calculation. The values of 
TABLE 5: Conductance components for InAs samples at temperatures $10 \mathrm{~K}$ and $70 \mathrm{~K}$.

\begin{tabular}{|c|c|c|c|c|c|c|c|}
\hline \multirow{3}{*}{ No. } & \multirow{3}{*}{ Parameter } & \multicolumn{3}{|c|}{$10 \mathrm{~K}$} & \multicolumn{2}{|c|}{$70 \mathrm{~K}$} & \multirow{3}{*}{$\begin{array}{l}\text { InAs } \\
4 \mu \mathrm{m}\end{array}$} \\
\hline & & InAs & InAs & InAs & InAs & InAs & \\
\hline & & $9 \mu \mathrm{m}$ & $4.7 \mu \mathrm{m}$ & $9 \mu \mathrm{m}$ & $4.7 \mu \mathrm{m}$ & $5.6 \mu \mathrm{m}$ & \\
\hline 1 & $n=n_{e}+x_{e}+n_{D 2}\left[\mathrm{~cm}^{-3}\right]$ & $3.8 \cdot 10^{15}$ & $7.9 \cdot 10^{15}$ & $2.7 \cdot 10^{15}$ & $6.62 \cdot 10^{15}$ & $4.52 \cdot 10^{16}$ & $4.085 \cdot 10^{17}$ \\
\hline 2 & $\mu_{n}\left[\mathrm{~cm}^{2} / \mathrm{V} \cdot \mathrm{s}\right]$ & 8000 & 3600 & 49000 & 33500 & 17350 & $*$ \\
\hline 3 & $\sigma_{n}[1 / \Omega \mathrm{cm}]$ & 4.9 & 4.56 & 21.17 & 35.53 & 125.63 & * \\
\hline 4 & $\sigma_{n} / \sigma_{n}$ & 1 & 1 & 1 & 1 & 1 & * \\
\hline 5 & $n_{e}\left[\mathrm{~cm}^{-3}\right]$ & $1.7 \cdot 10^{15}$ & $2.01 \cdot 10^{15}$ & $5.03 \cdot 10^{15}$ & $5.04 \cdot 10^{15}$ & $1.495 \cdot 10^{16}$ & $1.43 \cdot 10^{16}$ \\
\hline 6 & $\mu_{n_{e}}\left[\mathrm{~cm}^{2} / \mathrm{V} \cdot \mathrm{s}\right]$ & 7653 & 3320.15 & 49000 & 33153 & 23972.1 & $*$ \\
\hline 7 & $\sigma_{n_{e}}[1 / \Omega \mathrm{cm}]$ & 2.07 & 1.07 & 21.87 & 26.76 & 57.41 & $*$ \\
\hline 8 & $\sigma_{n_{e}} / \sigma_{n}$ & 0.426 & 0.234 & 1.03 & 0.753 & 0.46 & $*$ \\
\hline 9 & $x_{e}\left[\mathrm{~cm}^{-3}\right]$ & $2.17 \cdot 10^{15}$ & $5.94 \cdot 10^{15}$ & $-1.06 \cdot 10^{14}$ & $5.64 \cdot 10^{15}$ & $3.023 \cdot 10^{16}$ & $3.94 \cdot 10^{17}$ \\
\hline 10 & $\mu_{x_{e}}\left[\mathrm{~cm}^{2} / \mathrm{V} \cdot \mathrm{s}\right]$ & 19465 & 2286 & -1914 & 7536.31 & 28202.4 & * \\
\hline 11 & $\sigma_{x_{e}}[1 / \Omega \mathrm{cm}]$ & 6.76 & 2.175 & 0.033 & 6.81 & 136.58 & * \\
\hline 12 & $\sigma_{x_{e}} / \sigma_{n}$ & 1.4 & 0.48 & 0.0015 & 0.192 & 1.087 & * \\
\hline 13 & $n_{D 2}\left[\mathrm{~cm}^{-3}\right]$ & $-4.63 \cdot 10^{14}$ & $-2.45 \cdot 10^{14}$ & $-2.13 \cdot 10^{14}$ & $-1.69 \cdot 10^{14}$ & $-8.66 \cdot 10^{14}$ & $*$ \\
\hline 14 & $\mu_{D 2}\left[\mathrm{~cm}^{2} / \mathrm{V} \cdot \mathrm{s}\right]$ & -100 & -472.22 & -2740 & -1623.56 & 28518.2 & * \\
\hline 15 & $\sigma_{D 2}[1 / \Omega \mathrm{cm}]$ & 0.0074 & 0.0185 & 0.093 & 0.044 & -3.96 & * \\
\hline 16 & $\sigma_{D 2} / \sigma_{n}$ & 0.0015 & 0.00406 & 0.0044 & 0.0124 & 0.0315 & * \\
\hline 17 & $\Sigma n\left[\mathrm{~cm}^{-3}\right]$ & $3.407 \cdot 10^{15}$ & $7.705 \cdot 10^{15}$ & $4.7 \cdot 10^{15}$ & $1.05 \cdot 10^{16}$ & $4.43 \cdot 10^{16}$ & $4.083 \cdot 10^{17}$ \\
\hline 18 & $G_{n}[\mathrm{~S}]$ & $4.41 \cdot 10^{-3}$ & $2.14 \cdot 10^{-3}$ & $1.9 \cdot 10^{-2}$ & $1.67 \cdot 10^{-2}$ & $7.03 \cdot 10^{-2}$ & * \\
\hline 19 & $G_{n_{e}}[\mathrm{~S}]$ & $1.86 \cdot 10^{-3}$ & $5.03 \cdot 10^{-4}$ & $1.97 \cdot 10^{-2}$ & $1.26 \cdot 10^{-2}$ & $3.2 \cdot 10^{-2}$ & * \\
\hline 20 & $G_{x_{e}}[S]$ & $6.0 \cdot 10^{-3}$ & $1.02 \cdot 10^{-3}$ & $2.97 \cdot 10^{-5}$ & $3.2 \cdot 10^{-3}$ & $7.65 \cdot 10^{-2}$ & $*$ \\
\hline 21 & $G_{D 2}[\mathrm{~S}]$ & $6.66 \cdot 10^{-6}$ & $8.7 \cdot 10^{-6}$ & $8.37 \cdot 10^{-5}$ & $2.068 \cdot 10^{-5}$ & $-2.2 \cdot 10^{-3}$ & $*$ \\
\hline 22 & $\Sigma G_{n_{e}+x_{e}+n_{D 2}}[\mathrm{~S}]$ & $7.87 \cdot 10^{-3}$ & $1.53 \cdot 10^{-3}$ & $1.98 \cdot 10^{-2}$ & $1.58 \cdot 10^{-2}$ & $10.63 \cdot 10^{-2}$ & * \\
\hline 23 & $\Sigma G_{n_{e}+x_{e}+n_{D 2}} / G_{n}$ & 1.78 & 0.71 & 1.042 & 0.95 & 1.5 & $*$ \\
\hline
\end{tabular}

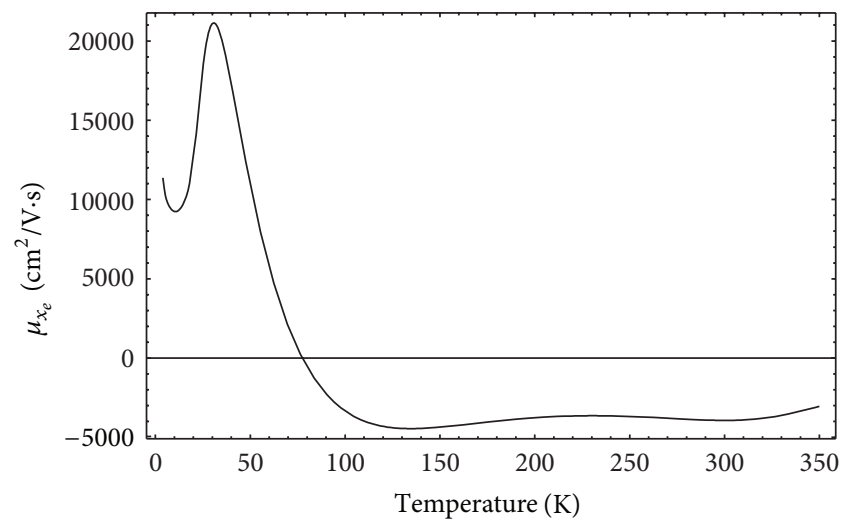

FIGURE 7: Mobility $\mu_{x_{e}}$ versus temperature, using the same calculation parameters used in Figures 3-5; negative values indicate hole behaviour (see Section 1-Models).

such charge carrier sources are chosen such that the calculated concentration curve approximates the experimental data $[3,26-32]$, as demonstrated for our samples (Table 2) by the example in Figure 3. We believe that the measured conductivity is affected by the presence of different donors in the semiconductor layers. Our model contains four charge carrier sources: two donors-one shallow $\left(N_{D 1}\right)$, located

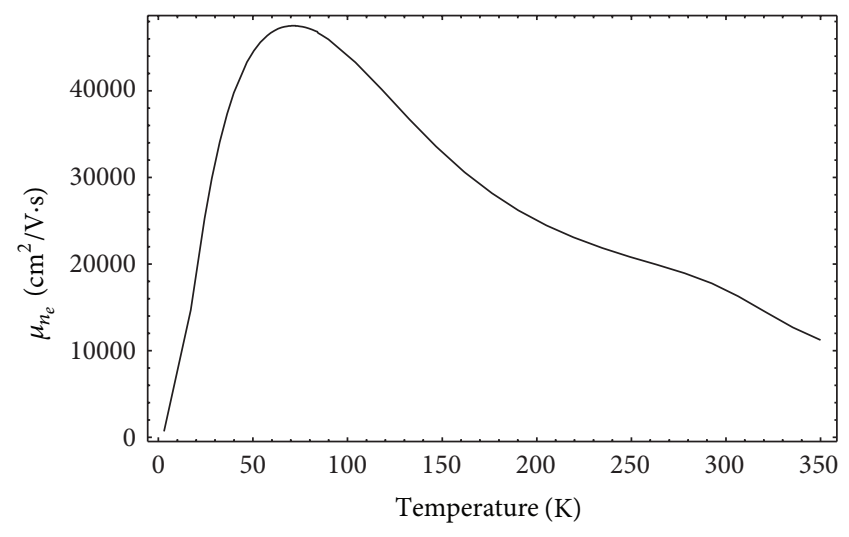

FIGURE 8: The electron mobility $\mu_{n_{e}}$ versus temperature, using the same calculation parameters used in Figures 3-5 (see Section 1Models).

under the conductivity band $\left(N_{D 1}\right)$, and one deeper $\left(N_{D 2}\right)$; one unknown $X$, which in our approach, can be donor-like or acceptor-like, depending on the temperature; and one acceptor $\left(N_{A}\right)$ as the source of holes. The elements $N_{D 1}$, $N_{D 2}$, and $N_{A}$ are located in the bulk of the epitaxial layer while $X$ is located at the interface between the substrate and the semiconductor within the first atomic layers (for 
TABLE 6: Conductance components for InAs samples at $200 \mathrm{~K}$.

\begin{tabular}{|c|c|c|c|c|c|}
\hline \multirow{3}{*}{ No. } & \multirow{3}{*}{ Parameter } & \multicolumn{4}{|c|}{$200 \mathrm{~K}$} \\
\hline & & InAs & InAs & InAs & InAs \\
\hline & & $9 \mu \mathrm{m}$ & $4.7 \mu \mathrm{m}$ & $5.6 \mu \mathrm{m}$ & $4 \mu \mathrm{m}$ \\
\hline 1 & $n=n_{e}+x_{e}+n_{D 2}\left[\mathrm{~cm}^{-3}\right]$ & $3.28 \cdot 10^{15}$ & $7.86 \cdot 10^{15}$ & $4.68 \cdot 10^{16}$ & $4.055 \cdot 10^{17}$ \\
\hline 2 & $\mu_{n}\left[\mathrm{~cm}^{2} / \mathrm{V} \cdot \mathrm{s}\right]$ & 24000 & 21900 & 16600 & 8700 \\
\hline 3 & $\sigma_{n}[1 / \Omega \mathrm{cm}]$ & 12.6 & 27.576 & 124.46 & 564. \\
\hline 4 & $\sigma_{n} / \sigma_{n}$ & 1 & 1 & 1 & 1 \\
\hline 5 & $n_{e}\left[\mathrm{~cm}^{-3}\right]$ & $4.0 \cdot 10^{15}$ & $8.12 \cdot 10^{15}$ & $3.41 \cdot 10^{16}$ & $9.88 \cdot 10^{16}$ \\
\hline 6 & $\mu_{n_{e}}\left[\mathrm{~cm}^{2} / \mathrm{V} \cdot \mathrm{s}\right]$ & 23570 & 29849.3 & 17016.3 & 10221 \\
\hline 7 & $\sigma_{n_{e}}[1 / \Omega \mathrm{cm}]$ & 15.65 & 27.12 & 92.96 & 162. \\
\hline 8 & $\sigma_{n_{e}} / \sigma_{n}$ & 1.24 & 0.983 & 0.75 & 0.286 \\
\hline 9 & $x_{e}\left[\mathrm{~cm}^{-3}\right]$ & $-7.5 \cdot 10^{14}$ & $-2.49 \cdot 10^{14}$ & $1.276 \cdot 10^{16}$ & $3.07 \cdot 10^{17}$ \\
\hline 10 & $\mu_{x_{e}}\left[\mathrm{~cm}^{2} / \mathrm{V} \cdot \mathrm{s}\right]$ & -4765 & -601.37 & 5692.03 & 31734 \\
\hline 11 & $\sigma_{x_{e}}[1 / \Omega \mathrm{cm}]$ & 0.57 & 0.024 & 11.63 & 1559 \\
\hline 12 & $\sigma_{x_{e}} / \sigma_{n}$ & 0.045 & 0.00087 & 0.093 & 2.76 \\
\hline 13 & $n_{D 2}\left[\mathrm{~cm}^{-3}\right]$ & $-9.0 \cdot 10^{13}$ & $5.88 \cdot 10^{14}$ & $-1.54 \cdot 10^{15}$ & ${ }^{*}$ \\
\hline 14 & $\mu_{D 2}\left[\mathrm{~cm}^{2} / \mathrm{V} \cdot \mathrm{s}\right]$ & -621 & 1506.67 & 5758.1 & * \\
\hline 15 & $\sigma_{D 2}[1 / \Omega \mathrm{cm}]$ & 0.009 & 0.142 & -1.42 & * \\
\hline 16 & $\sigma_{D 2} / \sigma_{n}$ & 0.0007 & 0.00515 & -0.0011 & * \\
\hline 17 & $\sum n\left[\mathrm{~cm}^{-3}\right]$ & $3.16 \cdot 10^{-15}$ & $8.46 \cdot 10^{15}$ & $4.5 \cdot 10^{16}$ & $4.058 \cdot 10^{17}$ \\
\hline 18 & $G_{n}[S]$ & $1.134 \cdot 10^{-2}$ & $1.3 \cdot 10^{-2}$ & $6.97 \cdot 10^{-2}$ & $22.56 \cdot 10^{-2}$ \\
\hline 19 & $G_{n_{e}}[S]$ & $1.41 \cdot 10^{-2}$ & $1.27 \cdot 10^{-2}$ & $5.2 \cdot 10^{-2}$ & $6.48 \cdot 10^{-2}$ \\
\hline 20 & $G_{x_{e}}[S]$ & $5.13 \cdot 10^{-4}$ & $1.13 \cdot 10^{-5}$ & $6.5 \cdot 10^{-3}$ & $62.36 \cdot 10^{-2}$ \\
\hline 21 & $G_{D 2}[\mathrm{~S}]$ & $8.1 \cdot 10^{-6}$ & $6.67 \cdot 10^{-5}$ & $-7.95 \cdot 10^{-4}$ & $*$ \\
\hline 22 & $\Sigma G_{n_{e}+x_{e}+n_{D 2}}[\mathrm{~S}]$ & $1.45 \cdot 10^{-2}$ & $1.28 \cdot 10^{-2}$ & $5.77 \cdot 10^{-2}$ & $68.84 \cdot 10^{-2}$ \\
\hline 23 & $\Sigma G_{n_{e}+x_{e}+n_{D 2}} / G_{n}$ & 1.28 & 0.98 & 0.83 & 3.05 \\
\hline
\end{tabular}

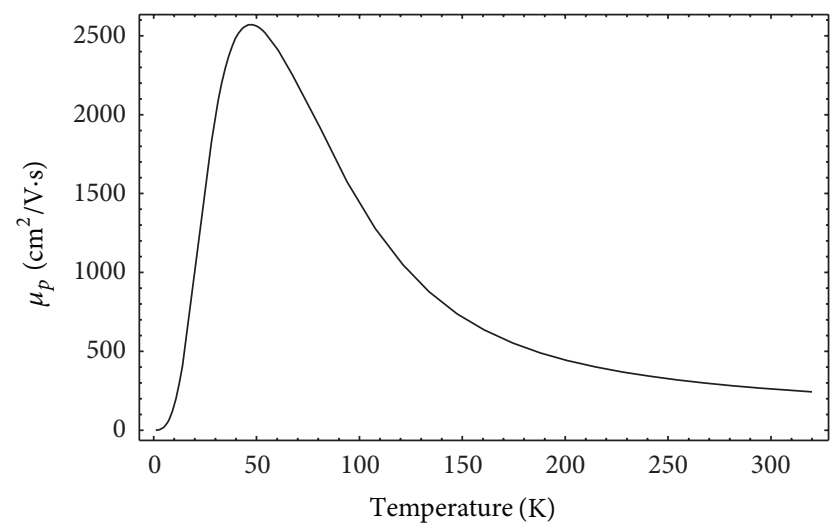

FIGURE 9: The hole mobility $\mu_{p}$ versus temperature, using the same calculation parameters used in Figures 3-5 (see Section 1-Models).

an explanation of this concept, see Section 1-Models). We numerically solve the neutrality equation, thereby combining the values of $N_{D 1}, N_{D 2}, X$, and $N_{A}$ together with their energies of activation, until we obtain the curves $n(T)$ or $n_{H}(T)$ lying as closely as possible to the experimental data at temperatures of 4-300 K. With values for $n(T)$ and $n_{H}(T)$ and using wellknown physical relations (see Section 1-Models), we can calculate all transport properties needed to determine the

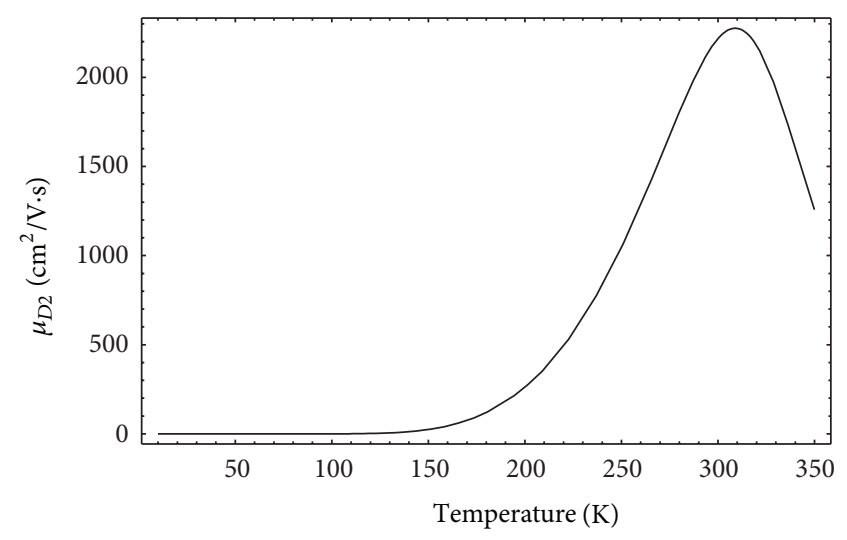

FIgURE 10: The electron mobility $\mu_{D 2}$ versus temperature, using the same calculation parameters used in Figures 3-5 (see Section 1Models).

conductance values. The negative values of the calculated transport parameters show that at the appropriate temperatures, charge carriers show hole-like properties. We suppose that our approach does not lose the physical interpretation of each step. The results were carefully analysed to ensure that the numerical calculations provided an accurate physical model of the studied effect. 
TABLE 7: Conductance components for InAs samples at $300 \mathrm{~K}$.

\begin{tabular}{|c|c|c|c|c|c|}
\hline \multirow{3}{*}{ No. } & \multirow{3}{*}{ Parameter } & \multicolumn{4}{|c|}{$300 \mathrm{~K}$} \\
\hline & & InAs & InAs & InAs & InAs \\
\hline & & $9 \mu \mathrm{m}$ & $4.7 \mu \mathrm{m}$ & $5.6 \mu \mathrm{m}$ & $4 \mu \mathrm{m}$ \\
\hline 1 & $n=n_{e}+x_{e}+n_{D 2}\left[\mathrm{~cm}^{-3}\right]$ & $4.18 \cdot 1015$ & $9.62 \cdot 10^{15}$ & $4.97 \cdot 10^{16}$ & $4.09 \cdot 10^{17}$ \\
\hline 2 & $\mu_{n}\left[\mathrm{~cm}^{2} / \mathrm{V} \cdot \mathrm{s}\right]$ & 16000 & 15800 & 14580 & 8400 \\
\hline 3 & $\sigma_{n}[1 / \Omega \mathrm{cm}]$ & 10.7 & 24.35 & 116.08 & 550 \\
\hline 4 & $\sigma_{n} / \sigma_{n}$ & 1 & 1 & 1 & 1 \\
\hline 5 & $n_{e}\left[\mathrm{~cm}^{-3}\right]$ & $5.62 \cdot 10^{15}$ & $8.13 \cdot 10^{15}$ & $4.27 \cdot 10^{16}$ & $1.45 \cdot 10^{17}$ \\
\hline 6 & $\mu_{n_{e}}\left[\mathrm{~cm}^{2} / \mathrm{V} \cdot \mathrm{s}\right]$ & 16240 & 15611 & 14565.7 & 9090 \\
\hline 7 & $\sigma_{n_{e}}[1 / \Omega \mathrm{cm}]$ & 14.6 & 20.33 & 99.64 & 211 \\
\hline 8 & $\sigma_{n_{e}} / \sigma_{n}$ & 1.36 & 0.835 & 0.858 & 0.38 \\
\hline 9 & $x_{e}\left[\mathrm{~cm}^{-3}\right]$ & $-1.43 \cdot 10^{15}$ & $-7.56 \cdot 10^{14}$ & $7.073 \cdot 10^{15}$ & $2.64 \cdot 10^{17}$ \\
\hline 10 & $\mu_{x_{e}}\left[\mathrm{~cm}^{2} / \mathrm{V} \cdot \mathrm{s}\right]$ & -4430 & -1109.67 & 2275.6 & 16535 \\
\hline 11 & $\sigma_{x_{e}}[1 / \Omega \mathrm{cm}]$ & 1.01 & 0.134 & 2.58 & 698 \\
\hline 12 & $\sigma_{x_{e}} / \sigma_{n}$ & 0.095 & 0.0055 & 0.022 & 1.27 \\
\hline 13 & $n_{D 2}\left[\mathrm{~cm}^{-3}\right]$ & $4.5 \cdot 10^{14}$ & $1.862 \cdot 10^{15}$ & $-1.51 \cdot 10^{15}$ & ${ }^{*}$ \\
\hline 14 & $\mu_{D 2}\left[\mathrm{~cm}^{2} / \mathrm{V} \cdot \mathrm{s}\right]$ & 1965 & 3636.22 & 2302.22 & $*$ \\
\hline 15 & $\sigma_{D 2}[1 / \Omega \mathrm{cm}]$ & 0.14 & 1.085 & -0.56 & * \\
\hline 16 & $\sigma_{D 2} / \sigma_{n}$ & 0.013 & 0.0445 & -0.048 & $*$ \\
\hline 17 & $\Sigma n\left[\mathrm{~cm}^{-3}\right]$ & $4.64 \cdot 10^{15}$ & $9.24 \cdot 10^{15}$ & $4.83 \cdot 10^{16}$ & $4.09 \cdot 10^{17}$ \\
\hline 18 & $G_{n}[\mathrm{~S}]$ & $9.63 \cdot 10^{-3}$ & $1.145 \cdot 10^{-2}$ & $6.5 \cdot 10^{-2}$ & $22.0 \cdot 10^{-2}$ \\
\hline 19 & $G_{n_{e}}[\mathrm{~S}]$ & $1.314 \cdot 10^{-2}$ & $9.55 \cdot 10^{-3}$ & $5.58 \cdot 10^{-2}$ & $8.44 \cdot 10^{-2}$ \\
\hline 20 & $G_{x_{e}}[S]$ & $9.09 \cdot 10^{-4}$ & $6.3 \cdot 10^{-5}$ & $1.44 \cdot 10^{-3}$ & $27.92 \cdot 10^{-2}$ \\
\hline 21 & $G_{D 2}[\mathrm{~S}]$ & $1.26 \cdot 10^{-4}$ & $5.1 \cdot 10^{-4}$ & $3.14 \cdot 10^{-4}$ & * \\
\hline 22 & $\Sigma G_{n_{e}+x_{e}+n_{D 2}}[\mathrm{~S}]$ & $1.417 \cdot 10^{-2}$ & $1.012 \cdot 10^{-2}$ & $5.75 \cdot 10^{-2}$ & $36.36 \cdot 10^{-2}$ \\
\hline 23 & $\Sigma G_{n_{e}+x_{e}+n_{D 2}} / G_{n}$ & 1.47 & 0.88 & 0.88 & 1.65 \\
\hline
\end{tabular}

TABLE 8: Scattering mechanism involved in calculation of InGaAs samples properties.

\begin{tabular}{|c|c|c|c|c|c|}
\hline No. & Parameter & $\begin{array}{c}\mathrm{In}_{0.53} \mathrm{Ga}_{0.47} \mathrm{As} \\
7 \mu \mathrm{m}\end{array}$ & $\begin{array}{c}\mathrm{In}_{0.53} \mathrm{Ga}_{0.47} \mathrm{As} \\
1.0 \mu \mathrm{m}\end{array}$ & $\begin{array}{c}\mathrm{In}_{0.53} \mathrm{Ga}_{0.47} \mathrm{As} \\
1.1 \mu \mathrm{m}\end{array}$ & $\begin{array}{c}\mathrm{In}_{0.53} \mathrm{Ga}_{0.47} \mathrm{As} \\
1.0 \mu \mathrm{m}\end{array}$ \\
\hline 1 & Mobility $\left[\mathrm{cm}^{2} / \mathrm{V} \cdot \mathrm{s}\right]$ & $\mu_{\mathrm{ii}}, \mu_{\mathrm{dp}}, \mu_{\mathrm{po}}, \mu_{\mathrm{pie}}$ & $\mu_{\mathrm{ii}}, \mu_{\mathrm{po}}, \mu_{\mathrm{sc}}$ & $\mu_{\mathrm{ii}}, \mu_{\mathrm{po}}, \mu_{\mathrm{sc}}$ & $\mu_{\mathrm{ii}}, \mu_{\mathrm{dp}}, \mu_{\mathrm{po}}, \mu_{\mathrm{pie}}, \mu_{\mathrm{sc}}$ \\
\hline
\end{tabular}

\section{Experimental}

The procedure for epitaxial layers of InAs is described as an example. The layers were obtained by molecular beam epitaxy (MBE) in a Riber 32P reactor on GaAs (001) substrates. The preparation conditions are presented in Table 3.

The layers exhibited very smooth surfaces and good crystallographic quality (RHEED results) and electrical properties (Hall measurements). The electrical properties are measured "ex situ" by the Hall effect (measurements currents of $10^{-4} \mathrm{~A}-10^{-3} \mathrm{~A}$ in a $0.6 \mathrm{~T}$ magnetic field). The Hall device had a square-shaped configuration with dimensions of 5 $\times 5 \mathrm{~mm}$. The ohmic contacts of the device were made of In $99 \%+$ Sn $1 \%$ alloy in a Bio-Rad Micro-science-Division (RC2400 Alloying Furnace). The linearity of the contacts characteristics were verified before each Hall effect measurement. All layers were intentionally undoped. The measured $n$-concentration of the wafers resulted from technical factors of preparation. $\mathrm{In}_{0.53} \mathrm{Ga}_{0.47}$ As layers were deposited by $\mathrm{MBE}$ on SI-InP and GaAs as well as SI-GaAs. We carried out the calculations as described previously [3] for a few samples of InAs, $\mathrm{In}_{0.53} \mathrm{Ga}_{0.47}$ As and GaAs with different charge carrier concentrations [3, 26-32]. The procedure consisted of the following steps:

(1) numerical solution of the neutrality equation,

(2) numerical calculation of the mobility of each component of the neutrality equation versus temperature;

(3) after determining the values for concentration and mobility versus temperature, we could obtain the conductivity values.

To validate the accuracy of the calculation, we compared the resistivity versus temperature, that is, calculated the curve using experimental data as in Figure 3 (resistivity was measured without a magnetic field; see Figure 4).

We present a more detailed example of the calculation for an InAs sample measuring $9.0 \mu \mathrm{m}$ thick (see Tables 2, 4, 5, 6, and 7, and all figures) [28]. The InAs samples have the lowest $E_{G}$ (Table 2). The results are presented in Figures 3, 4, 5, 6, 7, 8,9 , and 10 with the parameters used to solve the neutrality 
TABLE 9: Conductance components for InGaAs samples at temperatures of $10 \mathrm{~K}$ and $70 \mathrm{~K}$.

\begin{tabular}{|c|c|c|c|c|c|c|}
\hline \multirow{3}{*}{ No. } & \multirow{3}{*}{ Parameter } & \multirow{3}{*}{$\begin{array}{c}10 \mathrm{~K} \\
\mathrm{In}_{0.53} \mathrm{Ga}_{0.47} \mathrm{As} \\
7 \mu \mathrm{m}\end{array}$} & \multicolumn{4}{|c|}{$70 \mathrm{~K}$} \\
\hline & & & $\mathrm{In}_{0.53} \mathrm{Ga}_{0.47} \mathrm{As}$ & $\mathrm{In}_{0.53} \mathrm{Ga}_{0.47} \mathrm{As}$ & $\mathrm{In}_{0.53} \mathrm{Ga}_{0.47} \mathrm{As}$ & $\mathrm{In}_{0.53} \mathrm{Ga}_{0.47} \mathrm{As}$ \\
\hline & & & $7 \mu \mathrm{m}$ & $1.0 \mu \mathrm{m}$ & $1.1 \mu \mathrm{m}$ & $1.0 \mu \mathrm{m}$ \\
\hline 1 & $n=n_{e}+x_{e}+n_{D 2}\left[\mathrm{~cm}^{-3}\right]$ & $1 \cdot 10^{13}$ & $1.2 \cdot 10^{13}$ & $2.66 \cdot 10^{15}$ & $1.095 \cdot 10^{17}$ & $1.8 \cdot 10^{19}$ \\
\hline 2 & $\mu_{n}\left[\mathrm{~cm}^{2} / \mathrm{V} \cdot \mathrm{s}\right]$ & 6000 & 38000 & 48835 & 4086.1 & 1770.3 \\
\hline 3 & $\sigma_{n}[1 / \Omega \mathrm{cm}]$ & 0.01 & 0.073 & 20.8 & 71.6 & 50.98 \\
\hline 4 & $\sigma_{n} / \sigma_{n}$ & 1 & 1 & 1 & 1 & 1 \\
\hline 5 & $n_{e}\left[\mathrm{~cm}^{-3}\right]$ & $9.5 \cdot 10^{13}$ & $1.5 \cdot 10^{13}$ & $2.4 \cdot 10^{15}$ & $*$ & $*$ \\
\hline 6 & $\mu_{n_{e}}\left[\mathrm{~cm}^{2} / \mathrm{V} \cdot \mathrm{s}\right]$ & 815 & 37300 & 98000 & $*$ & * \\
\hline 7 & $\sigma_{n_{e}}[1 / \Omega \mathrm{cm}]$ & 0.0124 & 0.09 & 37.67 & $*$ & * \\
\hline 8 & $\sigma_{n_{e}} / \sigma_{n}$ & 1.24 & 1.22 & 1.8 & * & $*$ \\
\hline 9 & $x_{e}\left[\mathrm{~cm}^{-3}\right]$ & $2.0 \cdot 10^{12}$ & 0.0 & $4.92 \cdot 10^{14}$ & $*$ & * \\
\hline 10 & $\mu_{x_{e}}\left[\mathrm{~cm}^{2} / \mathrm{V} \cdot \mathrm{s}\right]$ & -730 & -7650 & -78000 & * & * \\
\hline 11 & $\sigma_{x_{e}}[1 / \Omega \mathrm{cm}]$ & -0.00023 & 0.0 & -6.15 & * & * \\
\hline 12 & $\sigma_{x_{e}} / \sigma_{n}$ & -0.023 & * & -0.295 & $*$ & $*$ \\
\hline 13 & $n_{D 2}\left[\mathrm{~cm}^{-3}\right]$ & 0.0 & $8 \cdot 10^{9}$ & $3.45 \cdot 10^{7}$ & $*$ & * \\
\hline 14 & $\mu_{D 2}\left[\mathrm{~cm}^{2} / \mathrm{V} \cdot \mathrm{s}\right]$ & -720 & -6710 & -53100 & * & * \\
\hline 15 & $\sigma_{D 2}[1 / \Omega \mathrm{cm}]$ & * & -0.000009 & $-2.93 \cdot 10^{-7}$ & * & * \\
\hline 16 & $\sigma_{D 2} / \sigma_{n}$ & $*$ & 0.000123 & $-1.41 \cdot 10^{-8}$ & $*$ & $*$ \\
\hline 17 & $\Sigma n\left[\mathrm{~cm}^{-3}\right]$ & $9.7 \cdot 10^{13}$ & $1.5 \cdot 10^{13}$ & $2.892 \cdot 10^{15}$ & $*$ & $*$ \\
\hline 18 & $G_{n}[\mathrm{~S}]$ & $7 \cdot 10^{-6}$ & $5.11 \cdot 10^{-5}$ & 0.00208 & $7.88 \cdot 10^{-3}$ & $5.098 \cdot 10^{-3}$ \\
\hline 19 & $G_{n_{e}}[S]$ & $8.68 \cdot 10^{-6}$ & $6.3 \cdot 10^{-5}$ & 0.0038 & * & $*$ \\
\hline 20 & $G_{x_{e}}[S]$ & $-1.61 \cdot 10^{-7}$ & 0 & $-6.15 \cdot 10^{-4}$ & * & * \\
\hline 21 & $G_{D 2}[\mathrm{~S}]$ & $*$ & * & $*$ & * & $*$ \\
\hline 22 & $\Sigma G_{n_{e}+x_{e}+n_{D 2}}[\mathrm{~S}]$ & $8.52 \cdot 10^{-6}$ & $6.3 \cdot 10^{-5}$ & 0.003185 & * & * \\
\hline 23 & $\Sigma G_{n_{e}+x_{e}+n_{D 2}} / G_{n}$ & 1.22 & 1.235 & 1.53 & $*$ & $*$ \\
\hline
\end{tabular}

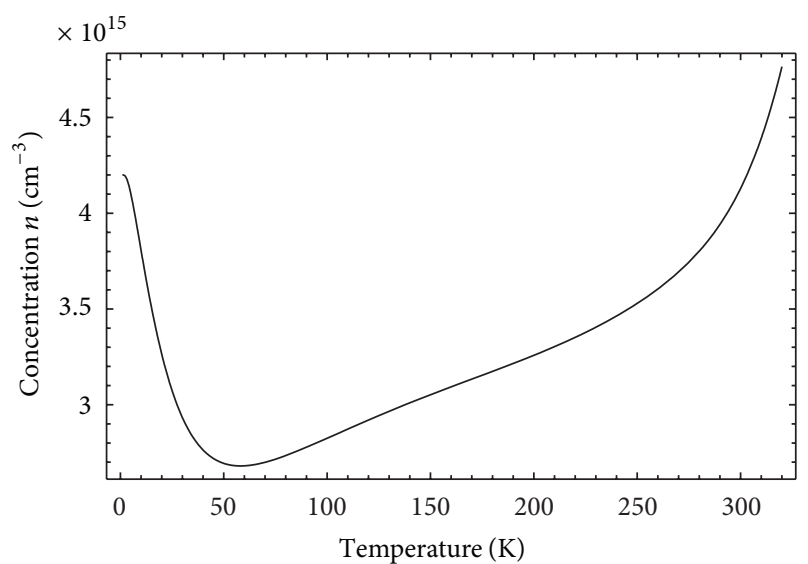

FIGURE 11: The $n$ concentration versus temperature, using the same calculation parameters used in Figures 3-5 (see Section 1-Models).

equation (according to $[3,32])$. In addition to the results presented in [28], we provide the Hall curves calculated by using the dependence $r_{H}=\left\langle\tau_{n, h}^{2}\right\rangle /\left\langle\tau_{n, h}\right\rangle^{2}$ and the calculated values of $n, n_{e}, x_{e}$ and $n_{D 2}$ versus temperature in Figures 11, 12, 13 , and 14 .

Figure 3 presents the results of solving the neutrality equation $[3,28,32]$ for the InAs sample. In this case,

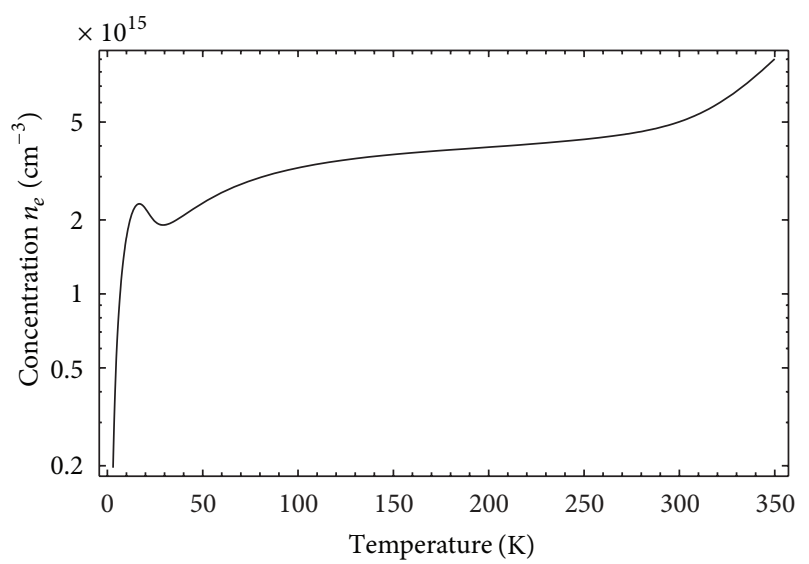

FIGURE 12: The $n_{e}$ concentration versus temperature, using the same calculation parameters used in Figures 3-5; (see Section 1-Models).

we used the metallurgical thickness of the epitaxial layer, which allowed us to calculate the conformity between the thickness values used to solve for the concentration and resistivity versus temperature. At low temperatures, there was an increase in concentration (Figure 3), which was probably due to the presence of impurity band conduction. The sample has an additional donor concentration $N_{D 2}$ at $0.13 \mathrm{eV}$ below 
TABLE 10: Conductance components for InGaAs samples at $200 \mathrm{~K}$.

\begin{tabular}{|c|c|c|c|c|c|}
\hline \multirow{3}{*}{ No. } & \multirow{3}{*}{ Parameter } & \multicolumn{4}{|c|}{$200 \mathrm{~K}$} \\
\hline & & $\mathrm{In}_{0.53} \mathrm{Ga}_{0.47} \mathrm{As}$ & $\mathrm{In}_{0.53} \mathrm{Ga}_{0.47} \mathrm{As}$ & $\mathrm{In}_{0.53} \mathrm{Ga}_{0.47} \mathrm{As}$ & $\mathrm{In}_{0.53} \mathrm{Ga}_{0.47} \mathrm{As}$ \\
\hline & & $7 \mu \mathrm{m}$ & $1.0 \mu \mathrm{m}$ & $1.1 \mu \mathrm{m}$ & $1 \mu \mathrm{m}$ \\
\hline 1 & $n=n_{e}+x_{e}+n_{D 2}\left[\mathrm{~cm}^{-3}\right]$ & $4.0 \cdot 10^{13}$ & $4.65 \cdot 10^{15}$ & $1.04 \cdot 10^{17}$ & $1.799 \cdot 10^{19}$ \\
\hline 2 & $\mu_{n}\left[\mathrm{~cm}^{2} / \mathrm{V} \cdot \mathrm{s}\right]$ & 19000 & 12810 & 8514 & 2010.5 \\
\hline 3 & $\sigma_{n}[1 / \Omega \mathrm{cm}]$ & 0.122 & 9.54 & 141.85 & 5794 \\
\hline 4 & $\sigma_{n} / \sigma_{n}$ & 1 & 1 & 1 & 1 \\
\hline 5 & $n_{e}\left[\mathrm{~cm}^{-3}\right]$ & $1.95 \cdot 10^{14}$ & $8.0 \cdot 10^{15}$ & $8.42 \cdot 10^{16}$ & $1.2 \cdot 10^{18}$ \\
\hline 6 & $\mu_{n_{e}}\left[\mathrm{~cm}^{2} / \mathrm{V} \cdot \mathrm{s}\right]$ & 18370 & 14400 & 8842 & 3045 \\
\hline 7 & $\sigma_{n_{e}}[1 / \Omega \mathrm{cm}]$ & 0.574 & 18.45 & 119.26 & 585 \\
\hline 8 & $\sigma_{n_{e}} / \sigma_{n}$ & 4.7 & 1.93 & 0.84 & 0.1 \\
\hline 9 & $x_{e}\left[\mathrm{~cm}^{-3}\right]$ & $-1.85 \cdot 10^{14}$ & $-2.7 \cdot 10^{15}$ & $2.53 \cdot 10^{16}$ & $1.68 \cdot 10^{19}$ \\
\hline 10 & $\mu_{x_{e}}\left[\mathrm{~cm}^{2} / \mathrm{V} \cdot \mathrm{s}\right]$ & -13400 & -6320 & 1643.3 & 35440 \\
\hline 11 & $\sigma_{x_{e}}[1 / \Omega \mathrm{cm}]$ & 0.4 & 2.73 & 6.66 & 95381 \\
\hline 12 & $\sigma_{x_{e}} / \sigma_{n}$ & 3.28 & 0.29 & 0.047 & 16.46 \\
\hline 13 & $n_{D 2}\left[\mathrm{~cm}^{-3}\right]$ & $3.6 \cdot 10^{13}$ & $5.3 \cdot 10^{13}$ & $1.38 \cdot 10^{16}$ & $*$ \\
\hline 14 & $\mu_{D 2}\left[\mathrm{~cm}^{2} / \mathrm{V} \cdot \mathrm{s}\right]$ & 64870 & -1840 & -882 & * \\
\hline 15 & $\sigma_{D 2}[1 / \Omega \mathrm{cm}]$ & 0.37 & -0.016 & -1.95 & * \\
\hline 16 & $\sigma_{D 2} / \sigma_{n}$ & 3.03 & -0.00164 & -0.014 & * \\
\hline 17 & $\Sigma n\left[\mathrm{~cm}^{-3}\right]$ & $4.6 \cdot 10^{13}$ & $5.35 \cdot 10^{15}$ & $1.233 \cdot 10^{17}$ & $1.8 \cdot 10^{19}$ \\
\hline 18 & $G_{n}[S]$ & $8.54 \cdot 10^{-5}$ & $9.54 \cdot 10^{-4}$ & $1.56 \cdot 10^{-2}$ & $57.94 \cdot 10^{-2}$ \\
\hline 19 & $G_{n_{e}}[\mathrm{~S}]$ & $4.02 \cdot 10^{-4}$ & $1.845 \cdot 10^{-3}$ & $1.31 \cdot 10^{-2}$ & $5.85 \cdot 10^{-2}$ \\
\hline 20 & $G_{x_{e}}[\mathrm{~S}]$ & $2.8 \cdot 10^{-4}$ & $2.73 \cdot 10^{-4}$ & $7.33 \cdot 10^{-4}$ & 9.538 \\
\hline 21 & $G_{D 2}[\mathrm{~S}]$ & $2.59 \cdot 10^{-4}$ & $-1.6 \cdot 10^{-6}$ & $-2.145 \cdot 10^{-4}$ & ${ }^{*}$ \\
\hline 22 & $\Sigma G_{n_{e}+x_{e}+n_{D 2}}[\mathrm{~S}]$ & $9.41 \cdot 10^{-4}$ & $2.12 \cdot 10^{-3}$ & $1.36 \cdot 10^{-2}$ & 9.59 \\
\hline 23 & $\Sigma G_{n_{e}+x_{e}+n_{D 2}} / G_{n}$ & 11.02 & 2.22 & 0.872 & 16.55 \\
\hline
\end{tabular}

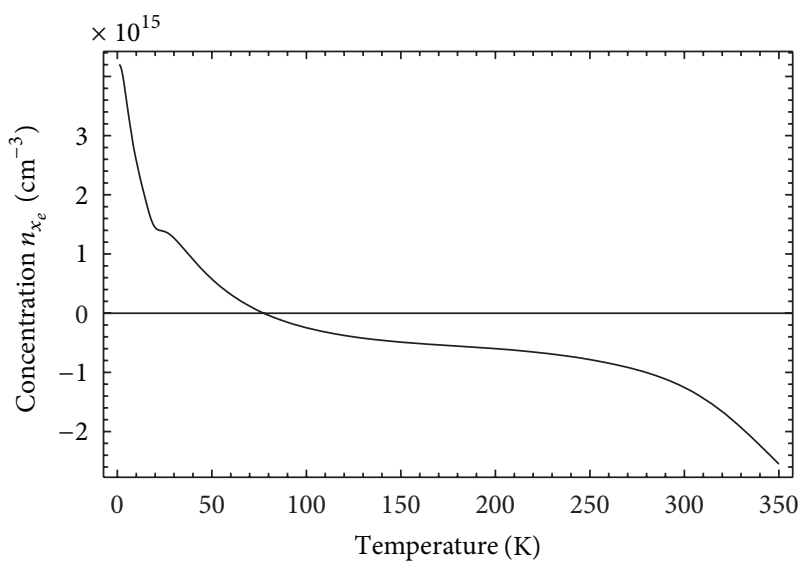

FIGURE 13: The $x_{e}$ concentration versus temperature, using the same calculation parameters used in Figures 3-5; concentration values below $0 x_{e}$ indicate hole-like behaviour (see Section 1-Models).

the bottom of the conduction band. This level is near $E_{G} / 2$ for InAs (Table 2). This conductivity concentration $(n)$ was used to calculate the resistivity-temperature (Figure 4) and the mobility-temperature curves (Figure 5). The calculated Hall concentration $n_{H}$ below $\sim 100 \mathrm{~K}$ lies distinctly under the experimental data.

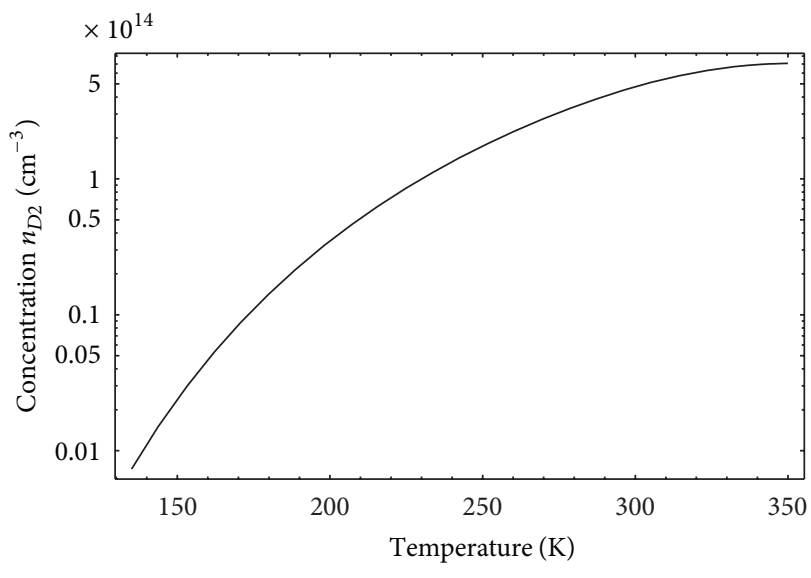

FIGURE 14: The $n_{D 2}$ concentration versus temperature, using the same calculation parameters used in Figures 3-5 (see Section 1Models).

We also verified the ratio of $n$ to the density of states value in the conductivity band versus temperature curve. This ratio determines the degeneration probability in the investigated sample. Unfortunately, below $\sim 15 \mathrm{~K}$, its value is greater than 1; therefore, all calculations below that temperature that use Boltzmann statistics are problematic (Figure 6). It must be 
TABLE 11: Conductance components for InGaAs samples at $300 \mathrm{~K}$.

\begin{tabular}{|c|c|c|c|c|c|}
\hline \multirow[b]{2}{*}{ No. } & \multirow[b]{2}{*}{ Parameter } & \multicolumn{4}{|c|}{$300 \mathrm{~K}$} \\
\hline & & $\begin{array}{c}\mathrm{In}_{0.53} \mathrm{Ga}_{0.47} \mathrm{As} \\
7 \mu \mathrm{m}\end{array}$ & $\begin{array}{c}\mathrm{In}_{0.53} \mathrm{Ga}_{0.47} \mathrm{As} \\
1.0 \mu \mathrm{m}\end{array}$ & $\begin{array}{c}\mathrm{In}_{0.53} \mathrm{Ga}_{0.47} \mathrm{As} \\
1.1 \mu \mathrm{m}\end{array}$ & $\begin{array}{c}\mathrm{In}_{0.53} \mathrm{Ga}_{0.47} \mathrm{As} \\
1.0 \mu \mathrm{m}\end{array}$ \\
\hline 1 & $n=n_{e}+x_{e}+n_{D 2}\left[\mathrm{~cm}^{-3}\right]$ & $1.2 \cdot 10^{14}$ & $6.75 \cdot 10^{15}$ & $1.13 \cdot 10^{17}$ & $1.796 \cdot 10^{19}$ \\
\hline 2 & $\mu_{n}\left[\mathrm{~cm}^{2} / \mathrm{V} \cdot \mathrm{s}\right]$ & 13000 & 8280 & 7310.5 & 1890 \\
\hline 3 & $\sigma_{n}[1 / \Omega \mathrm{cm}]$ & 0.25 & 8.95 & 132.2 & 5431 \\
\hline 4 & $\sigma_{n} / \sigma_{n}$ & 1 & 1 & 1 & 1 \\
\hline 5 & $n_{e}\left[\mathrm{~cm}^{-3}\right]$ & $1.6 \cdot 10^{14}$ & $1.0 \cdot 10^{16}$ & $9.76 \cdot 10^{16}$ & $1.55 \cdot 10^{18}$ \\
\hline 6 & $\mu_{n_{e}}\left[\mathrm{~cm}^{2} / \mathrm{V} \cdot \mathrm{s}\right]$ & 12970 & 8400 & 7477 & 2970 \\
\hline 7 & $\sigma_{n_{e}}[1 / \Omega \mathrm{cm}]$ & 0.33 & 13.4 & 116.8 & 732 \\
\hline 8 & $\sigma_{n_{e}} / \sigma_{n}$ & 1.32 & 1.5 & 0.88 & 0.13 \\
\hline 9 & $x_{e}\left[\mathrm{~cm}^{-3}\right]$ & $-1.45 \cdot 10^{14}$ & $-3.32 \cdot 10^{15}$ & $1.59 \cdot 10^{16}$ & $1.64 \cdot 10^{19}$ \\
\hline 10 & $\mu_{x_{e}}\left[\mathrm{~cm}^{2} / \mathrm{V} \cdot \mathrm{s}\right]$ & -6500 & -3230 & 875.6 & 25150 \\
\hline 11 & $\sigma_{x_{e}}[1 / \Omega \mathrm{cm}]$ & 0.15 & 1.72 & 2.23 & 66076 \\
\hline 12 & $\sigma_{x_{\rho}} / \sigma_{n}$ & 0.6 & 0.19 & 0.017 & 12.17 \\
\hline 13 & $n_{D 2}\left[\mathrm{~cm}^{-3}\right]$ & $1.2 \cdot 10^{14}$ & $6.9 \cdot 10^{14}$ & $1.72 \cdot 10^{16}$ & * \\
\hline 14 & $\mu_{D 2}\left[\mathrm{~cm}^{2} / \mathrm{V} \cdot \mathrm{s}\right]$ & 169360 & 25 & 443 & * \\
\hline 15 & $\sigma_{D 2}[1 / \Omega \mathrm{cm}]$ & 3.25 & 0.00276 & 1.22 & * \\
\hline 16 & $\sigma_{D 2} / \sigma_{n}$ & 13.0 & 0.0003 & 0.0092 & * \\
\hline 17 & $\Sigma n\left[\mathrm{~cm}^{-3}\right]$ & $1.35 \cdot 10^{14}$ & $7.37 \cdot 10^{15}$ & $1.135 \cdot 10^{17}$ & $1.795 \cdot 10^{19}$ \\
\hline 18 & $G_{n}[\mathrm{~S}]$ & $1.75 \cdot 10^{-4}$ & $8.95 \cdot 10^{-4}$ & $1.45 \cdot 10^{-2}$ & $54.31 \cdot 10^{-2}$ \\
\hline 19 & $G_{n_{e}}[\mathrm{~S}]$ & $2.31 \cdot 10^{-4}$ & $1.34 \cdot 10^{-3}$ & $1.28 \cdot 10^{-2}$ & $7.32 \cdot 10^{-2}$ \\
\hline 20 & $G_{x_{e}}[S]$ & $1.05 \cdot 10^{-4}$ & $1.72 \cdot 10^{-4}$ & $2.45 \cdot 10^{-4}$ & 6.608 \\
\hline 21 & $G_{D 2}[\mathrm{~S}]$ & $2.275 \cdot 10^{-3}$ & $2.76 \cdot 10^{-7}$ & $1.34 \cdot 10^{-4}$ & * \\
\hline 22 & $\Sigma G_{n_{\mathrm{e}}+x_{e}+n_{D^{2}}}[S]$ & $2.61 \cdot 10^{-3}$ & $1.35 \cdot 10^{-3}$ & $1.34 \cdot 10^{-2}$ & 6.68 \\
\hline 23 & $\Sigma G_{n_{n}+x_{x}+n_{n}} / G_{n}$ & 14.9 & 1.7 & 0.92 & 12.3 \\
\hline
\end{tabular}

TABLE 12: Scattering mechanism involved in calculation of GaAs samples properties.

\begin{tabular}{|c|c|c|c|c|c|}
\hline No. & Parameter & $\begin{array}{c}\text { GaAs } \\
4.25 \mu \mathrm{m}\end{array}$ & $\begin{array}{c}\text { GaAs } \\
1.7 \mu \mathrm{m}\end{array}$ & $\begin{array}{l}\mathrm{GaAs} \\
2.6 \mu \mathrm{m}\end{array}$ & $\begin{array}{c}\mathrm{GaAs} \\
2.4 \mu \mathrm{m}\end{array}$ \\
\hline 1 & $\begin{array}{l}\text { Mobility } \\
{\left[\mathrm{cm}^{2} / \mathrm{V} \cdot \mathrm{s}\right]}\end{array}$ & $\tau_{\mathrm{ii}}, \tau_{\mathrm{po}}, \tau_{\mathrm{sc}}$ & $\tau_{\mathrm{ii}}, \tau_{\mathrm{po}}, \tau_{\mathrm{sc}}$ & $\begin{array}{c}\tau_{\mathrm{ii}}, \tau_{\mathrm{po}}, \\
\tau_{\mathrm{sc}}\end{array}$ & $\begin{array}{c}\tau_{\mathrm{ii}}, \tau_{\mathrm{po}}, \\
\tau_{\mathrm{sc}}\end{array}$ \\
\hline
\end{tabular}

explained here that our charge transport model was very accurate only for the GaAs samples (Table 13) and the calculated values using the numerical method were sufficiently reliable. With respect to the two other semiconductors, our model was less accurate. The neutrality equation was properly solved for all investigated samples; therefore, we believe that the concentration values are consistent with the experimental results. However the InAs and InGaAs samples presented problems with the wafers exhibiting very high concentrations at temperatures below $200 \mathrm{~K}$ (InAs (Table 5) and InGaAs (Table 9)).

The resistivity curves (conductivity parameters) show very satisfactory behaviour. The calculated curve approximately agrees with the experimental data at all temperatures with some discrepancies only at temperatures below $15 \mathrm{~K}$. This disagreement is most marked below $100 \mathrm{~K}$, when the concentration is near $10 \%$ of the number of states in the conduction band (Figure 6). The great influence of the spacecharge scattering mechanism of the $N_{S} \cdot Q$ product (where $N_{S}$ is the density of space charge regions and $Q$ is their effective scattering cross-sectional area) must be underlined.

The Hall mobility measurements (as in Figure 5) lead to the conclusion that the theoretical $r_{H}$ disagrees with the real value $r_{H \text {,experimental }} \approx 1$ at lower temperatures (approximately as low as $\sim 100 \mathrm{~K}$ ).

We now present the results of mobility calculations of each component obtained after solution of the neutrality equation (Figures 7-10). At lower temperatures, donors named $D 2$ marginally influenced the total mobility; their numbers were so low that they could be omitted (they have important influence at temperatures $200-300 \mathrm{~K}$ ). It must be noted that calculated Hall effect curves (Figures 3 and 5 curves in red) lightly disagree with experimental points. The conductivity effect curves show much better compliance with the experiment, especially at low temperatures. Using the measurements procedure as described for the GaAs sample, we obtained results that allowed us to determine the components of conductivity for these samples (Table 2). The scattering mechanism used for this calculation and the pertinent values are shown: for InAs in Tables 4, 5, 6, and 7, for $\mathrm{In}_{0.53} \mathrm{Ga}_{0.47}$ As in Tables 8, 9, 10, and 11, and for GaAs in Tables 12 and 13. 
TABLE 13: Conductance components in GaAs.

\begin{tabular}{|c|c|c|c|c|c|c|c|c|c|c|}
\hline \multirow{3}{*}{ No. } & \multirow{3}{*}{ Parameter } & \multirow{3}{*}{$\begin{array}{c}10 \mathrm{~K} \\
\mathrm{GaAs} \\
1.7 \mu \mathrm{m}\end{array}$} & \multicolumn{4}{|c|}{$70 \mathrm{~K}$} & \multicolumn{4}{|c|}{$300 \mathrm{~K}$} \\
\hline & & & GaAs & GaAs & GaAs & GaAs & GaAs & GaAs & GaAs & GaAs \\
\hline & & & $4.25 \mu \mathrm{m}$ & $1.7 \mu \mathrm{m}$ & $2.6 \mu \mathrm{m}$ & $2.4 \mu \mathrm{m}$ & $4.25 \mu \mathrm{m}$ & $1.7 \mu \mathrm{m}$ & $2.6 \mu \mathrm{m}$ & $2.4 \mu \mathrm{m}$ \\
\hline 1 & $n=n_{e}+x_{e}+n_{D 2}\left[\mathrm{~cm}^{-3}\right]$ & $1.72 \cdot 10^{15}$ & $4.3 \cdot 10^{14}$ & $1.71 \cdot 10^{15}$ & $3.47 \cdot 10^{15}$ & $4.8 \cdot 10^{15}$ & $4.6 \cdot 10^{14}$ & $1.84 \cdot 10^{15}$ & $5.24 \cdot 10^{15}$ & $9.5 \cdot 10^{15}$ \\
\hline 2 & $\mu_{n}\left[\mathrm{~cm}^{2} / \mathrm{V} \cdot \mathrm{s}\right]$ & 8000 & 62000 & 48200 & 20730 & 19940 & 6000 & 8000 & 4315.4 & 3117.9 \\
\hline 3 & $\sigma_{n}[1 / \Omega \mathrm{cm}]$ & 2.2 & 4.27 & 13.5 & 11.52 & 15.33 & 0.45 & 2.36 & 3.62 & 4.74 \\
\hline 4 & $\sigma_{n} / \sigma_{n}$ & 1 & 1 & 1 & 1 & 1 & 1 & 1 & 1 & 1 \\
\hline 5 & $n_{e}\left[\mathrm{~cm}^{-3}\right]$ & $1.35 \cdot 10^{15}$ & $4.71 \cdot 10^{14}$ & $1.85 \cdot 10^{15}$ & $3.43 \cdot 10^{15}$ & $4.83 \cdot 10^{15}$ & $4.87 \cdot 10^{14}$ & $2.02 \cdot 10^{15}$ & $5.23 \cdot 10^{15}$ & $1.0 \cdot 10^{16}$ \\
\hline 6 & $\mu_{n_{e}}\left[\mathrm{~cm}^{2} / \mathrm{V} \cdot \mathrm{s}\right]$ & 7570 & 60793 & 48674 & 20730 & 19963 & 6141 & 7777 & 4323 & 3130 \\
\hline 7 & $\sigma_{n_{e}}[1 / \Omega \mathrm{cm}]$ & 1.64 & 4.58 & 14.42 & 11.4 & 15.45 & 0.478 & 2.52 & 3.62 & 5.01 \\
\hline 8 & $\sigma_{n_{e}} / \sigma_{n}$ & 0.744 & 1.07 & 1.07 & 0.99 & 1.008 & 1.06 & 1.07 & 1. & 1.056 \\
\hline 9 & $x_{e}\left[\mathrm{~cm}^{-3}\right]$ & $3.4 \cdot 10^{14}$ & $-4.31 \cdot 10^{13}$ & $-1.41 \cdot 10^{14}$ & $3.57 \cdot 10^{13}$ & $-3.25 \cdot 10^{13}$ & $-2.53 \cdot 10^{13}$ & $-2.17 \cdot 10^{14}$ & $1.2 \cdot 10^{13}$ & $-5.8 \cdot 10^{14}$ \\
\hline 10 & $\mu_{x_{e}}\left[\mathrm{~cm}^{2} / \mathrm{V} \cdot \mathrm{s}\right]$ & 1912 & -5273 & -3720 & 215.5 & -134.6 & -282.5 & -837.6 & 10 & -180 \\
\hline 11 & $\sigma_{x_{e}}[1 / \Omega \mathrm{cm}]$ & 0.104 & 0.0364 & 0.084 & 0.00123 & 0.0007 & 0.00114 & 0.03 & 0.00002 & 0.017 \\
\hline 12 & $\sigma_{x_{e}} / \sigma_{n}$ & 0.05 & 0.0085 & 0.0062 & 0.000107 & 0.000046 & 0.0025 & 0.0123 & 0.0000053 & 0.0036 \\
\hline 13 & $n_{D 2}\left[\mathrm{~cm}^{-3}\right]$ & $*$ & $4.37 \cdot 10^{8}$ & $1.43 \cdot 10^{10}$ & $3.6 \cdot 10^{9}$ & $1.55 \cdot 10^{13}$ & $4.98 \cdot 10^{13}$ & $2.20 \cdot 10^{14}$ & $1.0 \cdot 10^{15}$ & $4.0 \cdot 10^{15}$ \\
\hline 14 & $\mu_{D 2}\left[\mathrm{~cm}^{2} / \mathrm{V} \cdot \mathrm{s}\right]$ & * & 0.062 & 0.4 & 0.02 & 65 & 741.5 & 1085.65 & 1017 & 2273.6 \\
\hline 15 & $\sigma_{D 2}[1 / \Omega \mathrm{cm}]$ & * & * & ${ }^{*}$ & $*$ & 0.000161 & 0.0059 & 0.04 & 0.163 & 1.46 \\
\hline 16 & $\sigma_{D 2} / \sigma_{n}$ & * & * & * & * & 0.0000105 & 0.013 & 0.016 & 0.045 & 0.31 \\
\hline 17 & $\Sigma n\left[\mathrm{~cm}^{-3}\right]$ & $1.69 \cdot 10^{15}$ & $4.28 \cdot 10^{14}$ & $1.71 \cdot 10^{15}$ & $3.46 \cdot 10^{15}$ & $4.81 \cdot 10^{15}$ & $5.11 \cdot 10^{14}$ & $2.02 \cdot 10^{15}$ & $6.24 \cdot 10^{15}$ & $8.2 \cdot 10^{15}$ \\
\hline 18 & $G_{n}[\mathrm{~S}]$ & $3.74 \cdot 10^{-4}$ & $1.81 \cdot 10^{-3}$ & $2.295 \cdot 10^{-3}$ & $3.0 \cdot 10^{-3}$ & $3.68 \cdot 10^{-3}$ & $1.91 \cdot 10^{-4}$ & $4.01 \cdot 10^{-4}$ & $9.41 \cdot 10^{-4}$ & $1.14 \cdot 10^{-3}$ \\
\hline 19 & $G_{n_{e}}[\mathrm{~S}]$ & $2.79 \cdot 10^{-4}$ & $1.95 \cdot 10^{-3}$ & $2.45 \cdot 10^{-3}$ & $2.96 \cdot 10^{-3}$ & $3.71 \cdot 10^{-3}$ & $2.03 \cdot 10^{-4}$ & $4.28 \cdot 10^{-4}$ & $9.41 \cdot 10^{-4}$ & $1.20 \cdot 10^{-3}$ \\
\hline 20 & $G_{x_{e}}[\mathrm{~S}]$ & $1.77 \cdot 10^{-5}$ & $1.55 \cdot 10^{-5}$ & $1.43 \cdot 10^{-5}$ & $2.2 \cdot 10^{-7}$ & $1.7 \cdot 10^{-7}$ & $4.85 \cdot 10^{-7}$ & $5.1 \cdot 10^{-7}$ & $5.2 \cdot 10^{-9}$ & $4.08 \cdot 10^{-7}$ \\
\hline 21 & $G_{D 2}[\mathrm{~S}]$ & $*$ & $*$ & $*$ & $*$ & $3.86 \cdot 10^{-8}$ & $2.51 \cdot 10^{-6}$ & $6.8 \cdot 10^{-7}$ & $4.24 \cdot 10^{-5}$ & $3.5 \cdot 10^{-4}$ \\
\hline 22 & $\Sigma G_{n_{e}+x_{e}+n_{D 2}}[\mathrm{~S}]$ & $2.97 \cdot 10^{-4}$ & $1.965 \cdot 10^{-3}$ & $2.46 \cdot 10^{-3}$ & $2.96 \cdot 10^{-3}$ & $3.71 \cdot 10^{-3}$ & $2.06 \cdot 10^{-4}$ & $4.35 \cdot 10^{-4}$ & $9.83 \cdot 10^{-4}$ & $1.55 \cdot 10^{-3}$ \\
\hline 23 & $\Sigma G_{n_{e}+x_{e}+n_{D 2}} / G_{n}$ & 0.794 & 1.086 & 1.072 & 0.987 & 1.008 & 1.08 & 1.085 & 1.57 & 1.36 \\
\hline
\end{tabular}

${ }^{*}$ The values are so small that they can be omitted.

It may be concluded that with increased doping concentration of the InAs samples (Tables 5-7), fewer scattering mechanisms as determined by the calculation of the conductivity components apply (Table 4 ). The 23 rd row of each table shows the values of the ratio of the calculated conductance $\sum G_{n_{e}+x_{e}+D 2}$ used to measure $G_{n}$ for each structure. This ratio must be approximately equal to 1 . The actual values are presented in Figure 15. In Figures 16, 17, 18, and 19, the conductance components for a $4.7 \mu \mathrm{m}$ InAs sample at temperatures of $10 \mathrm{~K}, 70 \mathrm{~K}, 200 \mathrm{~K}$ and $300 \mathrm{~K}$ are shown. This sample exhibits $\sum G_{n_{e}+x_{e}+D 2} / G_{n}$ values of $0.71,0.95,0.98$, and 0.88 at $10 \mathrm{~K}, 70 \mathrm{~K}, 200 \mathrm{~K}$, and $300 \mathrm{~K}$, respectively. Also, six scattering mechanisms are involved in the calculation of the transport parameters (Table 4).

Figures 16-19 show that the changes in the conductance components of InAs changes are independent of temperature. The main part of the conductance is attributed to the $n_{e}$ part of the charge carriers. In Tables 5-7, some samples show a higher sum of the conductance components relative to the measured resultant conductance. We suppose that this is associated with the principles of the numerical calculation, which give approximate values. Also, the theoretical model that was used may be less accurate, due to the slightly higher doping of the semiconductor samples.
The scattering mechanism considered in for InGaAs calculation, the conductance components values, and the results of the calculated parameters are shown for 1.0-7 $\mu \mathrm{m}$ samples at temperatures of $70 \mathrm{~K}, 200 \mathrm{~K}$, and $300 \mathrm{~K}$ in Tables 8-11 and in Figures 21, 22, and 23.

The actual the values given in line 23 of Tables 9-11 (InGaAs) are presented in Figure 20. The values of $r_{H}$ were derived from experimental calculations and are equal to those for the samples with $n<1.10^{16} \mathrm{~cm}^{-3}$; however, for higher concentrations, the measured $r_{H}$ is equal to 1 and is different from that calculated according to equation $r_{H}=\left\langle\tau_{n, h}^{2}\right\rangle /\left\langle\tau_{n, h}\right\rangle^{2}$. The $\sum G_{n_{e}+x_{e}+D 2} / G_{n}$ ratio for InGaAs samples (Figure 20 ) is approximately equal to 1 at concentrations between $10^{15}$ and $10^{18}$, but for the samples with doping concentration of $\sim 10^{13}$ and $\sim 10^{19}$, our procedure yields erroneous results (Tables 911).

The scattering mechanism used for the GaAs calculations, the conductance components values, and the results of the calculated parameters are shown for $1.7-4.25 \mu \mathrm{m}$ samples at temperatures of $70 \mathrm{~K}, 200 \mathrm{~K}$, and $300 \mathrm{~K}$ in Tables 12 and 13, and those for the $1.7 \mu \mathrm{m}$ sample alone are shown in Figures 25,26 , and 27.

The actual values shown in line 23 of Table 13 (GaAs) are presented in Figure 24. In nearly all samples, the ratio 


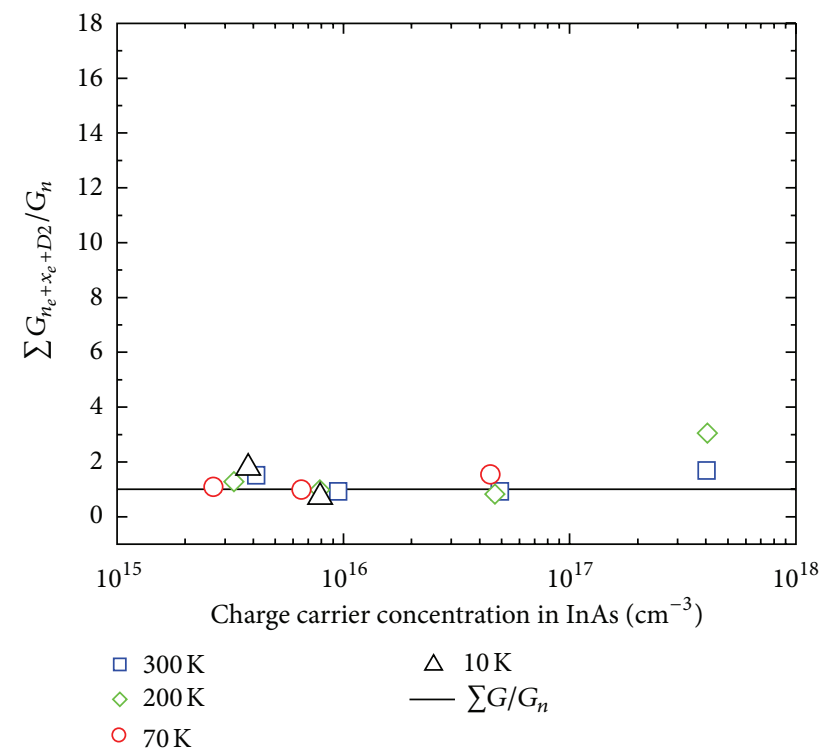

FIgURE 15: The actual values of the ratio $\sum G_{n_{e}+x_{e}+D 2} / G_{n}$ versus charge carriers concentration in the investigated InAs samples.

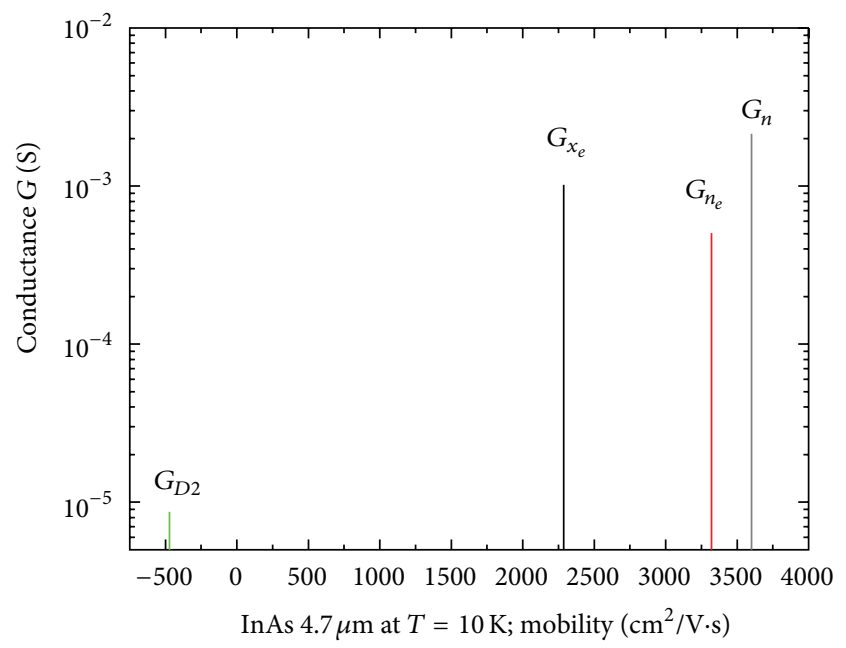

FIGURE 16: Conductance components for InAs $4.7 \mu \mathrm{m}$ at $10 \mathrm{~K}$.

$\sum G_{n_{e}+x_{e}+D 2} / G_{n}$ (Figure 24) is approximately equal to 1 . Only at $300 \mathrm{~K}$ is the concentration $n_{e}$ slightly higher in GaAs $4.25 \mu \mathrm{m}$ and GaAs $1.7 \mu \mathrm{m}$. From Figure 24, one can conclude that practically all GaAs samples present a $\sum G_{n_{e}+x_{e}+D 2} / G_{n}$ value equal to $\sim 1$.

\section{Conclusions}

As a followup to our previous papers [3,32], herein we present a potential application of the modelling and numerical simulation of material properties for three semiconductors InAs, $\mathrm{In}_{0.53} \mathrm{Ga}_{0.47}$ As, and GaAs as MBE layers by our MCF method using Hall structures. We have found three sources of current carriers, $n_{e}, n_{x_{e}}$, and $n_{D 2}$, whose sum should

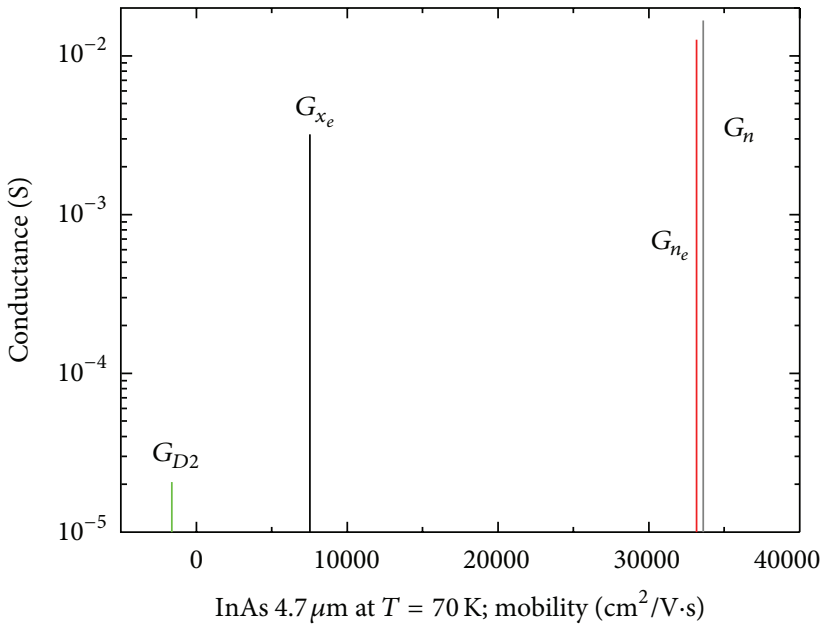

Figure 17: Conductance components for InAs $4.7 \mu \mathrm{m}$ at $70 \mathrm{~K}$.

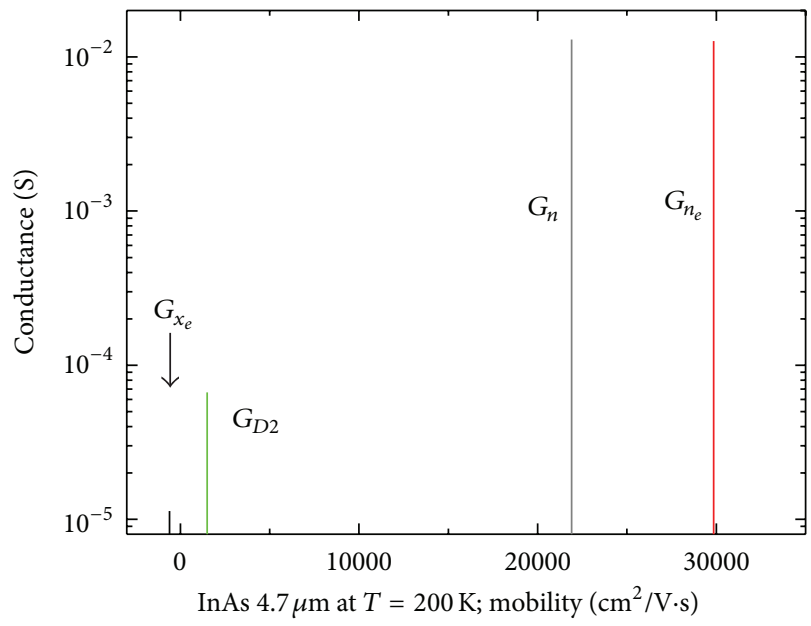

FIGURE 18: Conductance components for InAs $4.7 \mu \mathrm{m}$ at $200 \mathrm{~K}$.

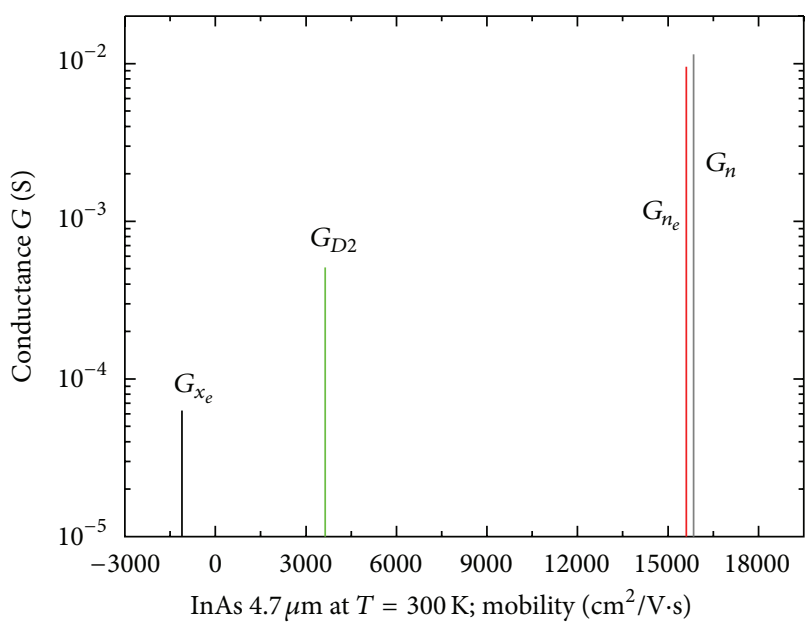

FIgURE 19: Conductance components for InAs $4.7 \mu \mathrm{m}$ at $300 \mathrm{~K}$. 


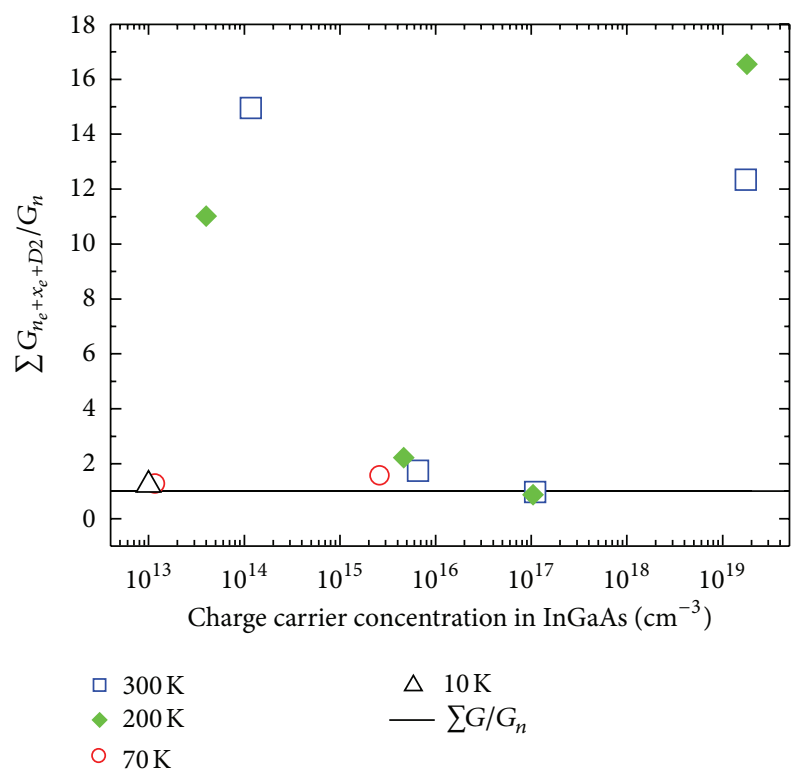

FIgURE 20: The actual values of the ratio $\sum G_{n_{e}+x_{e}+D 2} / G_{n}$ versus charge carriers concentration in the investigated InGaAs samples.

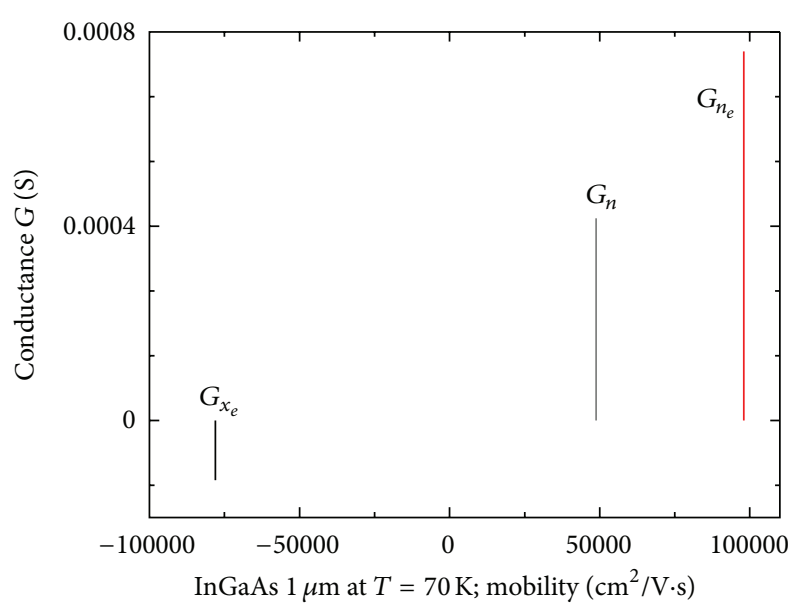

FIGURE 21: Conductance components for InGaAs $1.0 \mu \mathrm{m}$ at $70 \mathrm{~K}$.

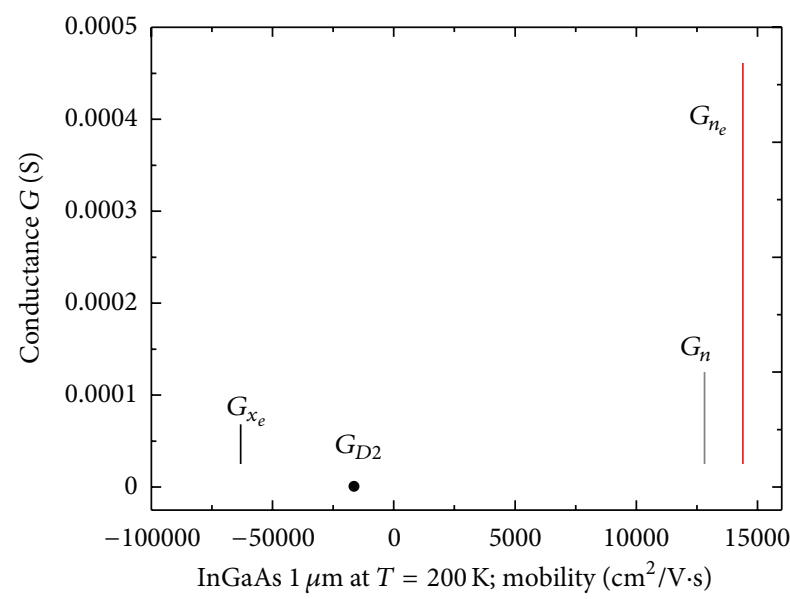

FIGURE 22: Conductance components for InGaAs $1.0 \mu \mathrm{m}$ at $200 \mathrm{~K}$.

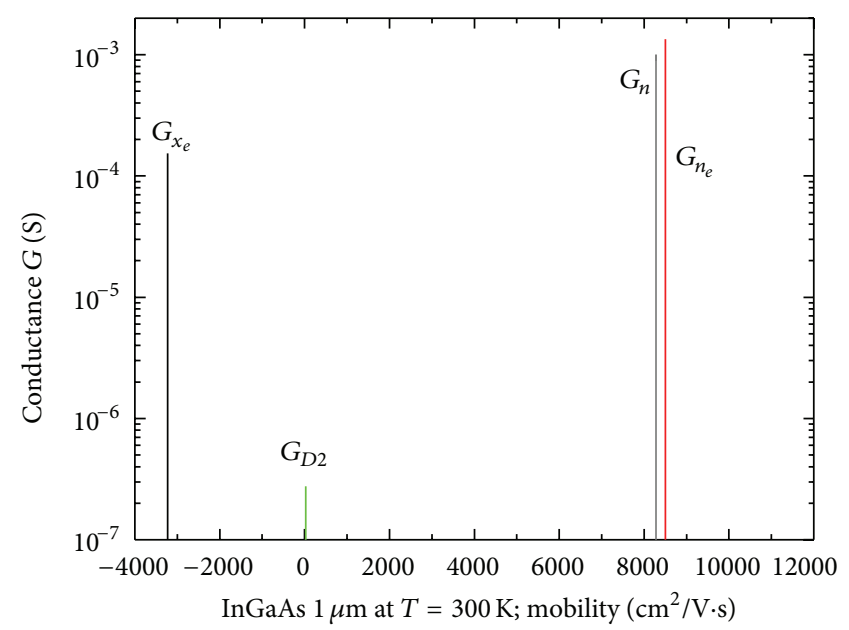

Figure 23: Conductance components for InGaAs $1.0 \mu \mathrm{m}$ at $300 \mathrm{~K}$.

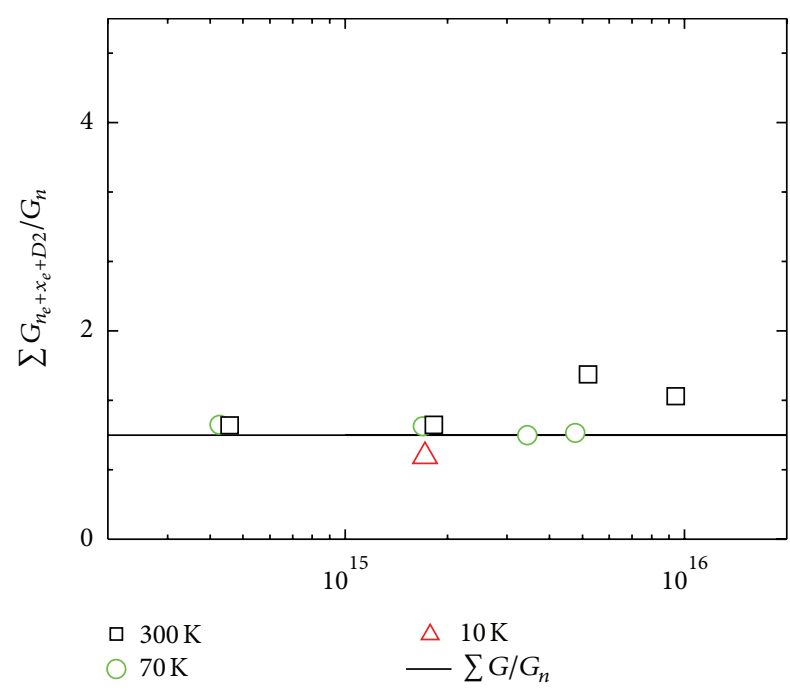

FIGURE 24: The actual values of the ratio $\sum G_{n_{e}+x_{e}+D 2} / G_{n}$ versus charge carrier concentration in the investigated $\mathrm{GaAs}$ sample.

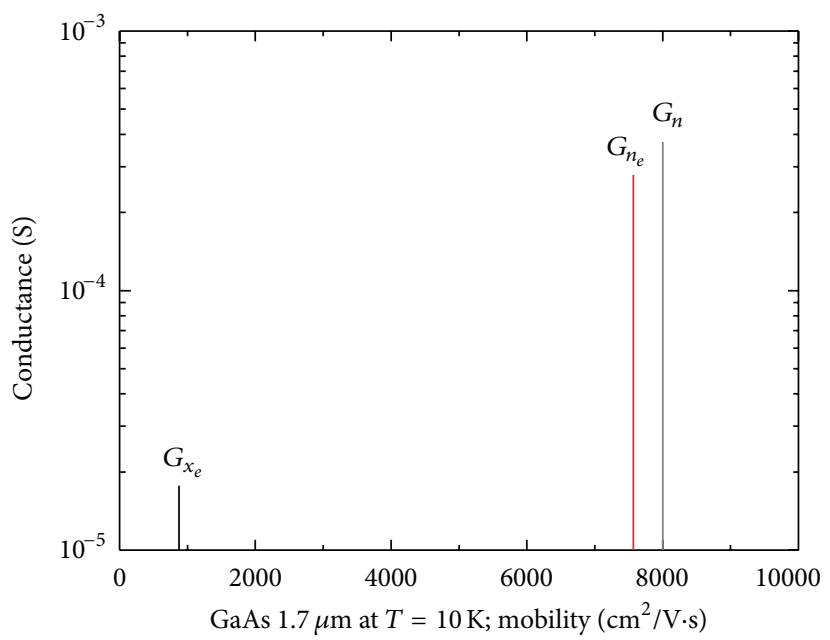

FIgURE 25: Conductance components for GaAs $1.7 \mu \mathrm{m}$ at $10 \mathrm{~K}$. 


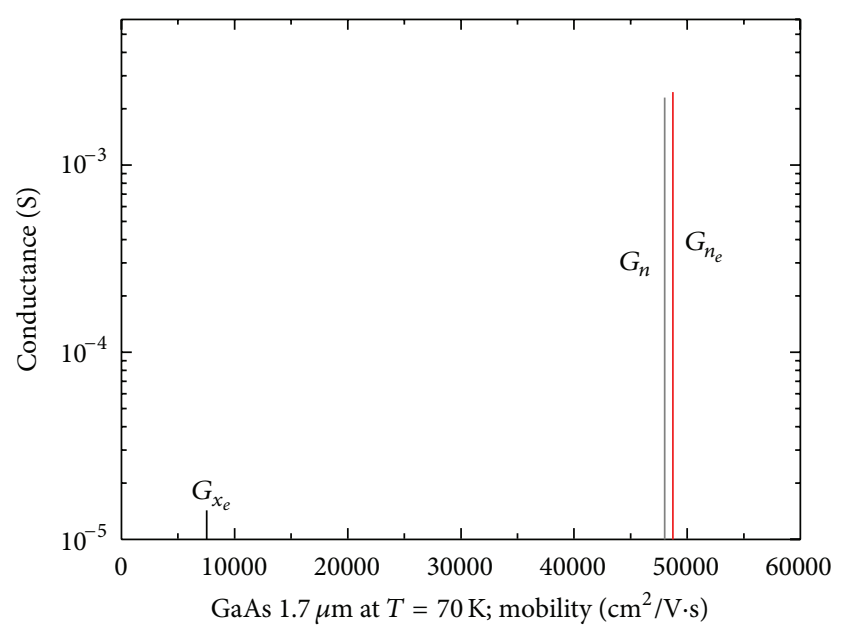

FIgUre 26: Conductance components for GaAs $1.7 \mu \mathrm{m}$ at $70 \mathrm{~K}$.

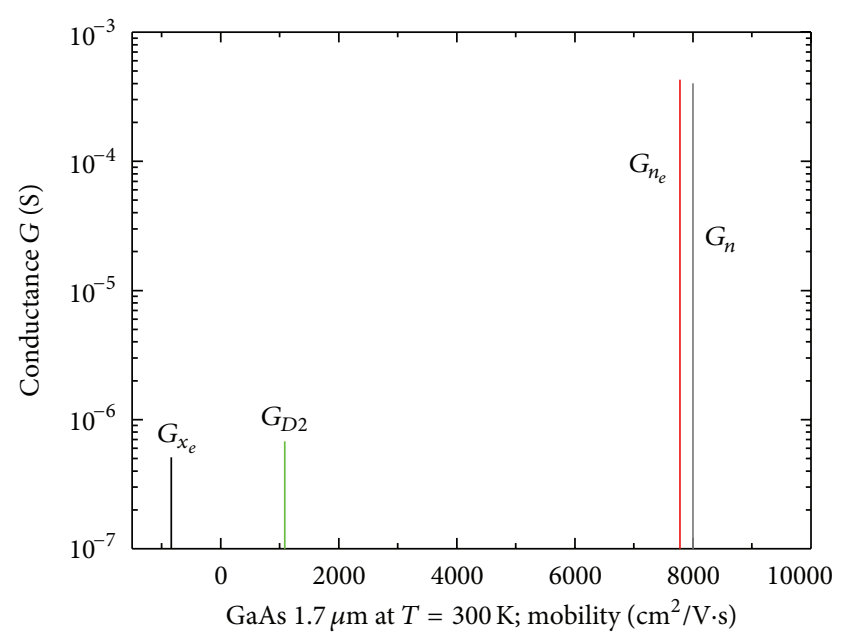

FIGURE 27: Conductance components for GaAs $1.7 \mu \mathrm{m}$ at $300 \mathrm{~K}$.

theoretically be $n$. We obtained the best results of numerical simulation for GaAs samples; for InAs and $\operatorname{In}_{0.53} \mathrm{Ga}_{0.47}$ As, our method is limited. The use of different scattering mechanisms (Tables 4, 8, and 12) allows for a more exhaustive description of conduction in the investigated MBE layers. We believe, nevertheless, that, in this paper, we demonstrate a novel application of the solution of the neutrality equation and relaxation time approximation by numerical calculations. It is also clear to us that some improvement in the theory of conduction in thin semiconductor layers formed by MBE is required.

The scattering mechanisms used to calculate the charge transport properties (Tables 4,8 , and 12) indicate that the crystallographic quality of samples composed of the same material may vary. Only the GaAs samples (Table 13) present the same value for the scattering mechanisms $\left(\tau_{\mathrm{ii}}, \tau_{\mathrm{po}}, \tau_{\mathrm{sc}}\right)$ in all of the investigated samples. The purest samples are those that have the largest thickness. Such behaviour agrees with our previous observations [3, 27-32].
The presented method for the numerical modelling and calculation of conductance components allows us to determine what parameters affect the total conductance. We calculated only the charge transport properties of carriers that originate from donors and acceptors using the solution of the neutrality equation. This solution is obtained such that the concentration versus temperature curve satisfactorily lies on the experimental data. The same applies to the resistivity and mobility. These three dependences underline the reliability of our calculations. In conclusion, we assume that our modelling method allows for the determination of the conductivity components strictly connected with experiments in relatively weak magnetic fields.

\section{Acknowledgments}

The author is indebted to Maciej Bugajski, Prof. and Janusz Kaniewski, DSc for their many helpful discussions. Special thanks are given to Tomasz Przesławski, PhD for the fruitful measurement results provided and Kazimierz Reginski, DSc for the samples preparation. The calculations are performed using math.8 at ICM Interdisciplinary Centre for Mathematical and Computational Modeling at University of Warsaw owing Calculation Grant G51-5.

\section{References}

[1] J. D. Grange, E. H. C. Parker, and R. M. King, "Relationship of MBE growth parameters with the electrical properties of thin (100) InAs epilayers," Journal of Physics D, vol. 12, no. 9, pp. 16011612, 1979.

[2] P. N. Fawcett, B. A. Joyce, X. Zhang, and D. W. Pashley, "Interface structure of InAs grown on GaAs(001) surfaces by molecular beam epitaxy," Journal of Crystal Growth, vol. 116, no. 1-2, pp. 81-86, 1992.

[3] A. Wolkenberg, T. Przeslawski, J. Kaniewski, E. Kowalczyk, and K. Reginski, "Conductivity analysis of n-GaAs molecular beam epitaxial layers using multicarrier fitting," Journal of Applied Physics, vol. 99, no. 9, Article ID 093708, 2006.

[4] M. C. Gold and D. A. Nelson, "Variable magnetic field Hall effect measurements and analysis of high purity, $\mathrm{Hg}$ vacancy (p-type)," Journal of Vacuum Science \& Technology, vol. 4, pp. 2040-2046, 1986.

[5] J. R. Meyer, C. A. Hoffman, F. J. Bartoli, D. A. Arnold, S. Sivananthan, and J. P. Fauri, "Methods for magnetotransport characterization of IR detector materials," Semiconductor Science and Technology, vol. 8, pp. 805-823, 1993.

[6] W. A. Beck and J. R. Anderson, "Determination of electrical transport properties using a novel magnetic field-dependent Hall technique," Journal of Applied Physics, vol. 62, no. 2, pp. 541-554, 1987.

[7] A. C. Beer, Galvanomagnetic Effects in Semiconductors, vol. 4 of Solid state physics: Supplement, 1963.

[8] Z. Dziuba, "Magnetotransport characterization of GaAs," Acta Physica Polonica, vol. 80, pp. 827-830, 1991.

[9] Z. Dziuba and M. Górska, "Analysis of the electrical conduction using an iterative method," Journal de Physique III, vol. 2, no. 1, pp. 99-110, 1992.

[10] J. Antoszewski, D. J. Seymour, L. Faraone, J. R. Meyer, and C. A. Hoffman, "Magneto-transport characterization using 
quantitative mobility-spectrum analysis," Journal of Electronic Materials, vol. 24, no. 9, pp. 1255-1262, 1995.

[11] Z. Dziuba, J. Antoszewski, J. M. Dell et al., "Magnetic field dependent Hall data analysis of electron transport in modulation-doped AlGaN/GaN heterostructures," Journal of Applied Physics, vol. 82, no. 6, pp. 2996-3002, 1997.

[12] J. R. Meyer, C. A. Hoffman, J. Antoszewski, and L. Faraone, "Quantitative mobility spectrum analysis of multicarrier conduction in semiconductors," Journal of Applied Physics, vol. 81, no. 2, pp. 709-713, 1997.

[13] D. K. Schroder, Semiconductor Material and Device Characterization, Wiley Interscience, 2006.

[14] H. M. Lin, Y. L. Chen, J. Yang et al., "Synthesis and characterization of core-shell GaP@GaN and GaN@GaP nanowires," Nano Letters, vol. 3, no. 4, pp. 537-541, 2003.

[15] A. Amtout, S. Raghavan, P. Rotella, G. Von Winckel, A. Stintz, and S. Krishna, "Theoretical modeling and experimental characterization of InAs/InGaAs quantum dots in a well detector," Journal of Applied Physics, vol. 96, no. 7, pp. 3782-3786, 2004.

[16] G. Brammertz, H. C. Lin, K. Martens et al., "Capacitancevoltage characterization of GaAs-Oxide interfaces," Journal of the Electrochemical Society, vol. 155, no. 12, pp. H945-H950, 2008.

[17] H. F. Chiu, Y. S. Chang, J. Y. Wu et al., "Very high deposition rate of a-Si:H thin films by ECRCVD," ECS Transactions, vol. 34, pp. 1065-1070, 2011.

[18] F. A. Ali, P. Pattnaik, and S. K. Kamilla, "Characterisation of bulk GaSb using TCAD," in Proceedings of the 2nd IEEE Students' Technology Symposium (TechSym '11), pp. 211-213, Kharagpur, India, January 2011.

[19] Q. Gao, H. H. Tan, H. E. Jackson et al., "Growth and properties of III-V compound semiconductor heterostructure nanowires," Semiconductor Science and Technology, vol. 26, no. 1, Article ID 014035, 2011.

[20] H. Arabshahi, "A new calculation method for thermal and electical characterization in CdTe and CdSe semiconductors," International Journal of Physical Sciences, vol. 6, no. 13, pp. 31553161, 2011.

[21] J. S. Kim, D. G. Seiler, and W. F. Tseng, "Multicarrier characterization method for extracting mobilities and carrier densities of semiconductors from variable magnetic field measurements," Journal of Applied Physics, vol. 73, no. 12, pp. 8324-8335, 1993.

[22] S. Kiatgamolchai, M. Myronov, O. A. Mironov, V. G. Kantser, E. H. C. Parker, and T. E. Whall, "Mobility spectrum computational analysis using a maximum entropy approach," Physical Review E, vol. 66, no. 3, Article ID 036705, 9 pages, 2002.

[23] D. Chrastina, J. P. Hague, and D. R. Leadley, "Application of Bryan's algorithm to the mobility spectrum analysis of semiconductor devices," Journal of Applied Physics, vol. 94, pp. 6583-6591, 2003.

[24] I. Vurgaftman, J. R. Meyer, C. A. Hoffman et al., "Improved quantitative mobility spectrum analysis for hall characterization," Journal of Applied Physics, vol. 84, no. 9, pp. 4966-4973, 1998.

[25] J. Antoszewski, L. Faraone, I. Vurgaftman, J. R. Meyer, and C. A. Huffman, "Application of quantitative mobility-spectrum analysis to multilayer HgCdTe structures," Journal of Electronic Materials, vol. 33, no. 6, pp. 673-683, 2004.

[26] A. Wolkenberg and T. Przesławski, "Application of galvanomagnetic measurements in temperature range $70-300 \mathrm{~K}$ to $\mathrm{MBE}$
GaAs layers characterization," in Metal/Nonmetal Microsystems: Physics, Technology, and Applications, vol. 2780 of Proceedings of SPIE, pp. 170-182, 1995.

[27] A. Wolkenberg, T. Przesławski, J. Kaniewski, J. Ba̧k-Misiuk, and K. Regiński, "Thickness dependence of the structural and electrical properties of InAs layers epitaxially grown by MBE on GaAs (001)," Materials Science and Engineering B, vol. 77, pp. 250-254, 2000.

[28] T. Przesławski, A. Wolkenberg, K. Regiński, J. Kaniewski, and J. Bak-Misiuk, "Growth and transport properties of relaxed epilayers of InAs on GaAs," Thin Solid Films, vol. 367, no. 1-2, pp. 232-234, 2000.

[29] A. Wolkenberg, T. Przesławski, K. Regiński, and J. Kaniewski, "Electrical charge transport of n-InAs epitaxial films on GaAs," Materials Science and Engineering B, vol. 90, no. 1-2, pp. 176-179, 2002.

[30] A. Wolkenberg, T. Przesławski, J. Kaniewski, and K. Regiński, "Experimental confirmation by galvanomagnetic methods of a complex transport model in $\operatorname{In}_{0.53} \mathrm{Ga}_{0.47}$ As layers deposited by MBE on SI-InP," Journal of Physics and Chemistry of Solids, vol. 64, no. 1, pp. 7-14, 2003.

[31] A. Wolkenberg, T. Przesławski, J. Kaniewski, and K. Reginski, "A method for calculating the conductivity mobility spectrum using multi-carrier fitting," Materials Science and Engineering $B$, vol. 110, no. 1, pp. 79-86, 2004.

[32] A. Wolkenberg and T. Przesławski, "Parametry galwanomagnetyczne i przewodnościowe warstw epitaksjalnych MBE $\mathrm{z}$ InAs/GaAs, $\mathrm{In}_{0,53} \mathrm{Ga}_{0,47}$ As/InP i GaAs/GaAs," Elektronika, vol. 48, pp. 26-38, 2007. 

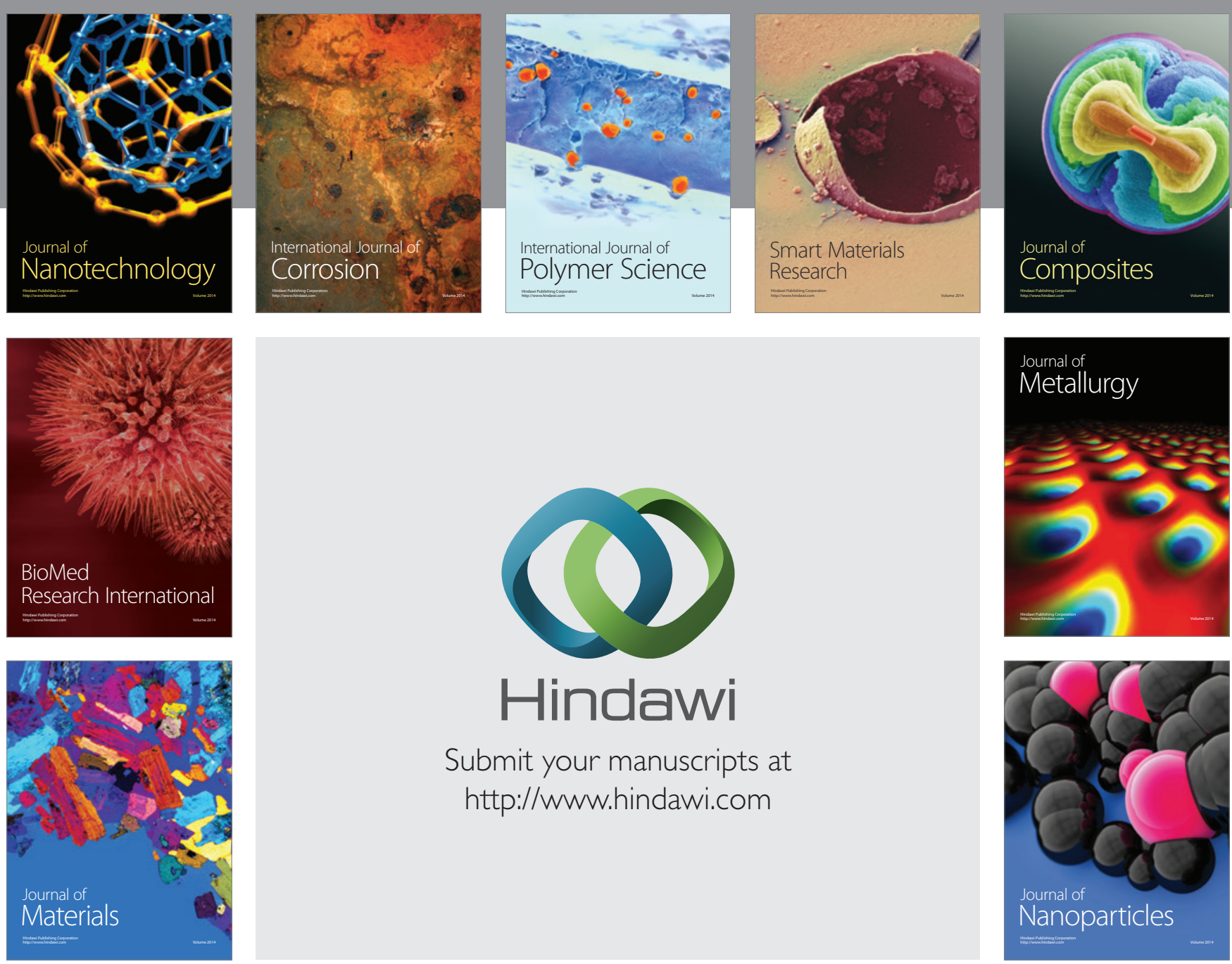

Submit your manuscripts at http://www.hindawi.com
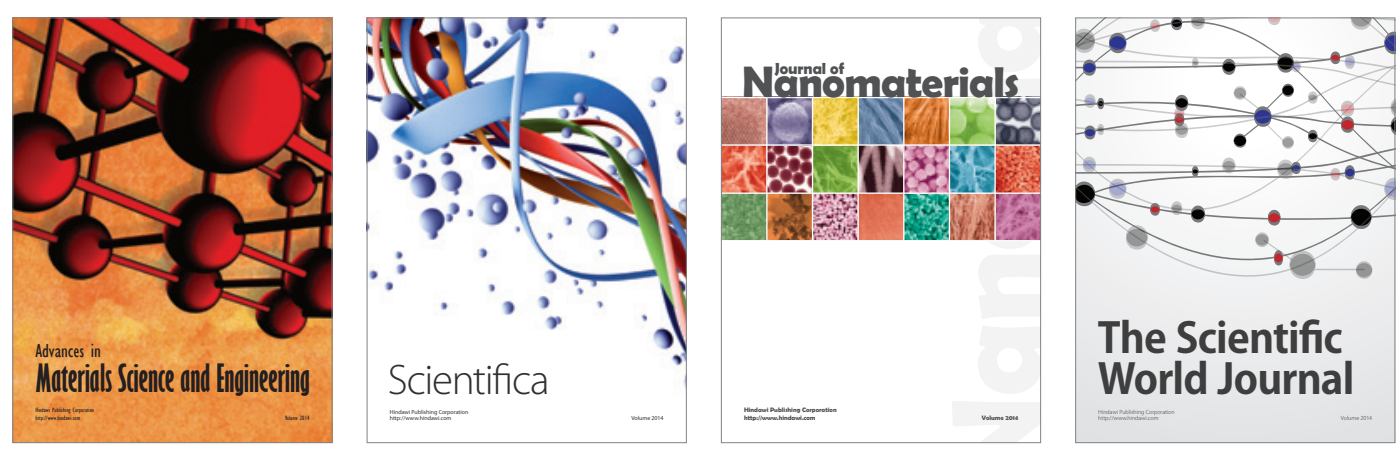

\section{The Scientific World Journal}
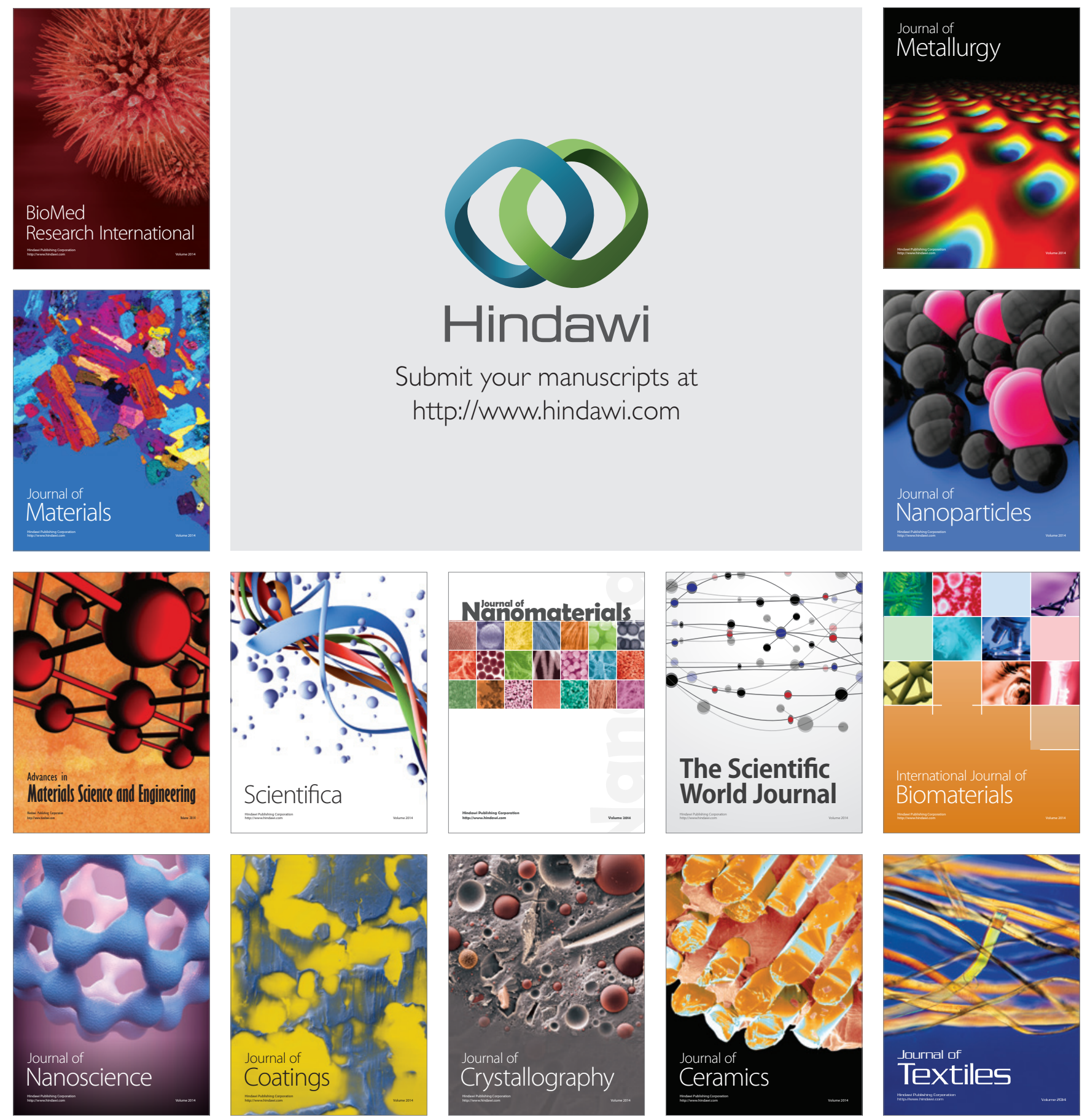